\title{
Feature Based Mobile Phone Rating Using Sentiment Analysis and Machine Learning Approaches
}

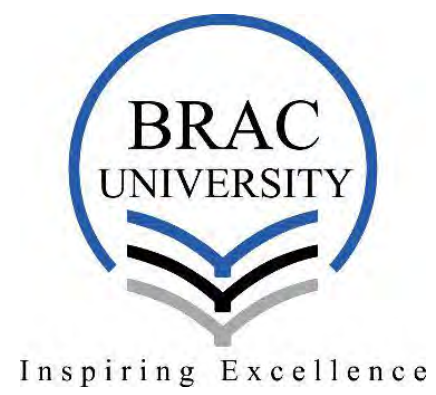

Thesis submitted in partial fulfillment of the requirement for the degree of Bachelor of Computer Science and Engineering

\section{SUBMITED BY}

Abdullahil Kafi

Md. Shaikh Ashikul Alam

Sayeed Bin Hossain

Siam Bin Awal

\section{SUPERVISOR}

Hossain Arif

Assistant Professor

Department of Computer Science and Engineering 


\section{DECLARATION}

We, hereby declare that this thesis is based on the results we obtained from our work. Due acknowledgement has been made in the text to all other material used. This thesis, neither in whole nor in part, has been previously submitted by anyone to any other university or institute for the award of any degree.

Signature of the Supervisor

\section{Hossain Arif}

Assistance Professor

Department of Computer Science and

Engineering

BRAC University
Signature of Authors

Abdullahil Kafi - 14301019

Md. Shaikh Ashikul Alam - 14301017

Sayeed Bin Hossain - 14301061

Siam Bin Awal - 14301040 


\begin{abstract}
This project proposes a model of sentiment analysis of different features of different company's mobile sets and rating them overall. Customers before buying a phone check reviews to get a better understanding of the device and this project derives an optimum solution for this. In this model, every feature of a mobile phone is rated based on public opinion and an overall rating for every type. Amazon is one of the largest internet retailer, which makes way for most public reviews on their products and so we collected data for sentiment analysis from amazon. We pre-processed the gathered data to a supervised form and chose the most common features from train data. In our model, Naïve Bayes, Support Vector Machine, Logistic Regression, Stochastic Gradient Descent and Random Forest algorithms were used to compare performance. These classification algorithms were trained with the training data and tested with test dataset to determine the accuracy of the classifiers. Our model provides an average polarity of each features and an average polarity of the mobile phone which will give a rating of the device, thus assisting the customers to choose the best according to their desire. This project can work as an assistant for the customers to determine their device following the opinion of the other users of the device.
\end{abstract}




\section{ACKNOWLEDGEMENT}

Firstly, we would like to thank our Almighty for enabling us to conduct our research, give our best efforts and conclude it.

Secondly, we would like to thank our supervisor Hossain Arif Sir for his feedback, support, guidance and contribution in conducting the research and preparation of the report. He encouraged us to conduct the research, guided us and always was present to offer any help we could ask for. We are grateful to him for his excellent supervision and effective guidance to successfully conduct our research.

We extend our gratitude to our parents and friends, who helped us with kindness and inspiration and with their suggestions. We would also like to acknowledge our fellow researchers whose informative suggestions and numerous resources aided us to achieve our goal.

Last but not the least, we thank BRAC University for providing us the opportunity of conducting this research and for giving us the chance to complete our Bachelor degree. 


\section{TABLE OF CONTENTS}

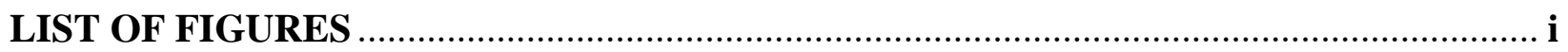

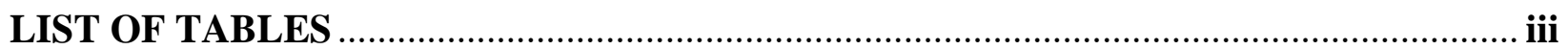

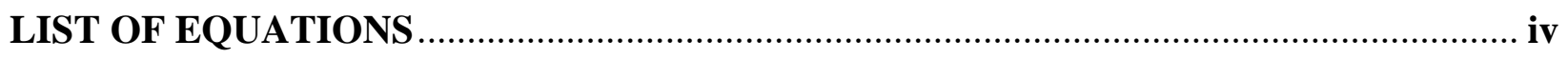

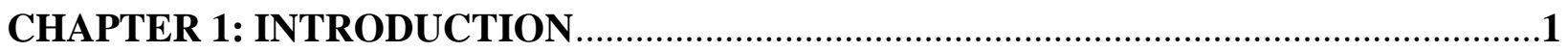

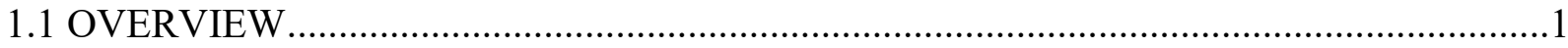

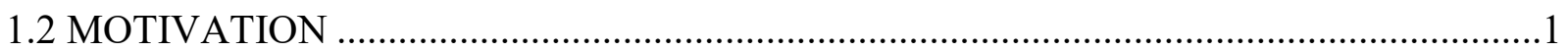

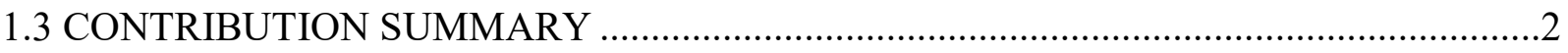

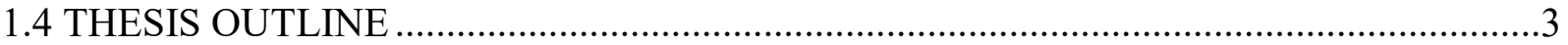

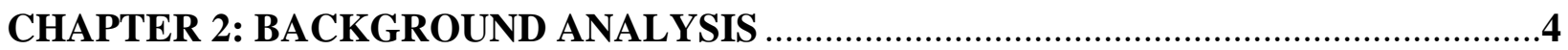

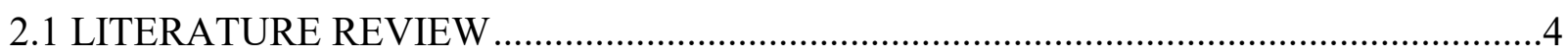

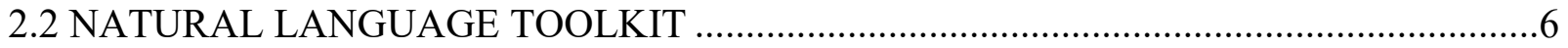

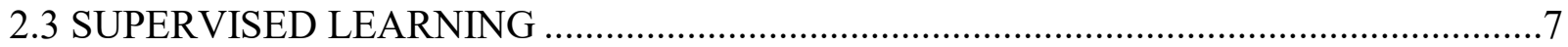

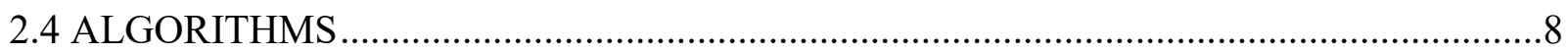

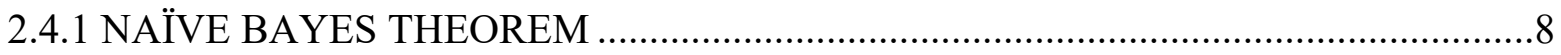

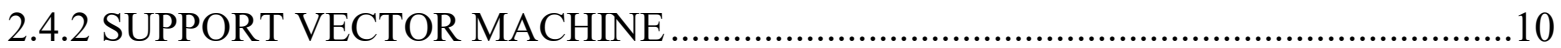

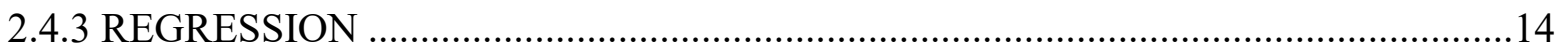

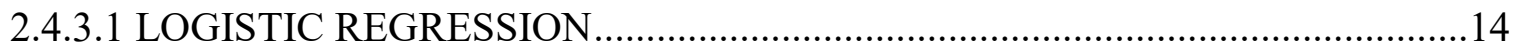

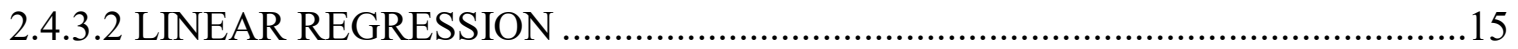

2.4.4 STOCHASTIC GRADIENT DESCENT (SGD) ……….........................................16

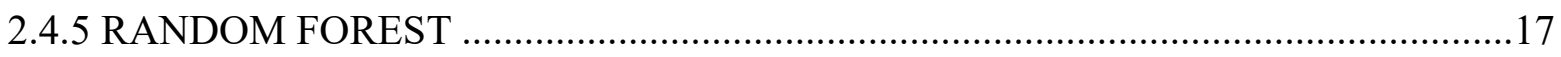

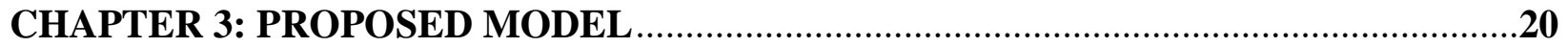

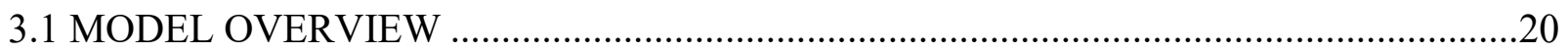

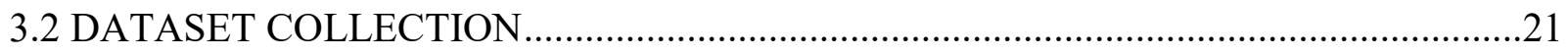

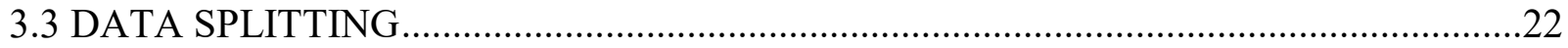

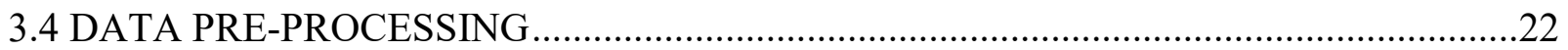

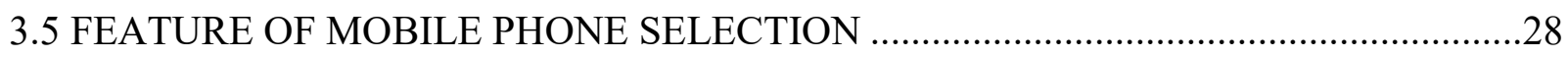

3.6 TRAINING ALGORITHMS AND TESTING ALGORITHM .........................................29

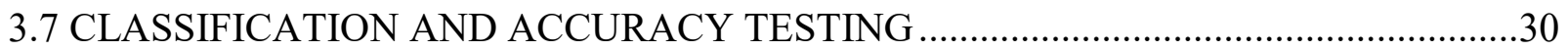

3.8 SENTIMENT POLARITY OF EACH FEATURES AND THE REVIEW ..........................35

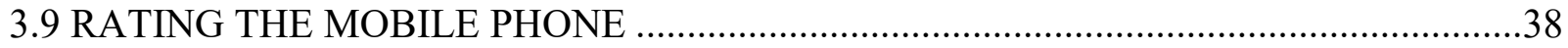


CHAPTER 4: EXPERIMENTAL SETUP \& ANALYSIS ...........................................41

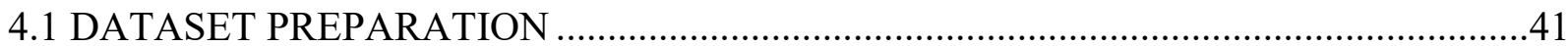

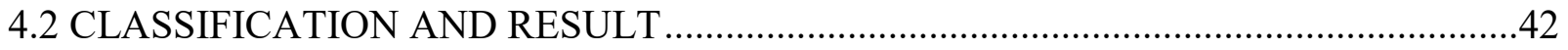

4.3 SENTIMENT ANALYSIS ON REVIEW .............................................................45

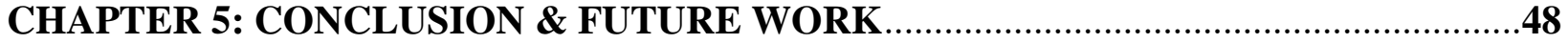

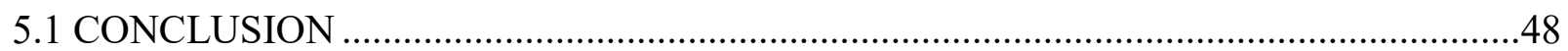

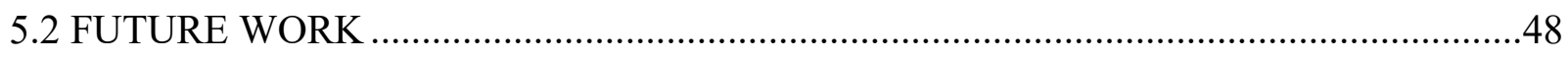

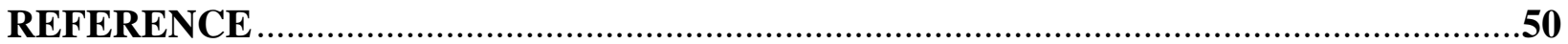




\section{LIST OF FIGURES}

Fig.1 TYPES OF NAÏVE BAYES CLASSIFICATION .............................................. 10

Fig.2 SVM CLASSIFICATIONS OF TWO CLASSES................................................. 11

Fig.3 SVM CLASSIFICATION USING HYPER-LINE ................................................ 12

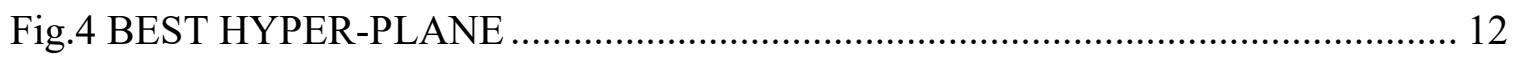

Fig.5 SVM CLASSIFICATIONS WITH JUMBLED DATA ......................................... 13

Fig.6 SVM CLASSIFICATION IN 3D DIMENSION................................................. 13

Fig.7 TYPES OF SVM CLASSIFICATION ............................................................. 14

Fig.8 PARAMETERS OF REGRESSION MODEL ....................................................... 15

Fig.9 BLOCK DIAGRAM OF THE PROPOSED MODEL ………………………….... 21

Fig.10 BLOCK DIAGRAM OF TEXT PRE-PROCESSING ………………………..... 23

Fig.11 TOKENIZED VERSION OF THE REVIEW …………………........................ 24

Fig.12 RESULT AFTER APPLYING STEMMING …………..................................... 25

Fig.13 RESULT OF APPLYING LEMMATIZATION................................................ 26

Fig.14 RESULT OF REMOVING STOP WORDS ……………………………….... 27

Fig.15 RESULT OF APPLYING POS TAGGING.................................................... 27

Fig.16 MOST COMMON FEATURES OF TRAIN DATA ………………………….... 28

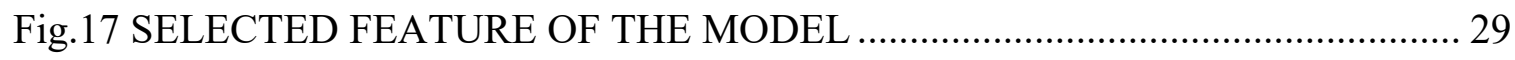

Fig.18 ACCURACY OF ALL CLASSIFIERS ............................................................. 35

Fig.19 SELECTION OF ADJECTIVE AND SPECIFICATION (FEATURE) ............... 36

Fig.20 RESULT OF SENTIMENT ANALYSIS......................................................... 37

Fig.21 RESULT OF POS TAGGING TO THE REVIEW ............................................. 37

Fig.22 RESULT OF FEATURE BASED SENTIMENT ANALYSIS ……………….... 38

Fig.23 FINAL RESULT OF SENTIMENT ANALYSIS ............................................... 39

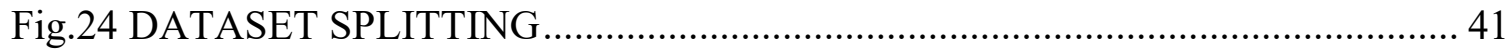

Fig.25 COMPARISON BETWEEN NAÏVE BAYES CLASSIFIERS ............................ 42

Fig.26 COMPARISON BETWEEN SVC CLASSIFIERS ............................................ 43

Fig.27 COMPARISON BETWEEN CLASSIFIERS ………........................................... 43

Fig.28 ACCURACY COMPARISON BETWEEN ALL CLASSIFIERS AND VOTED

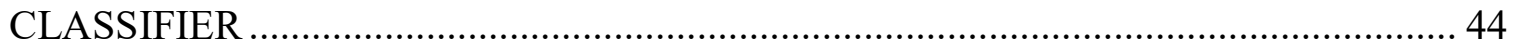




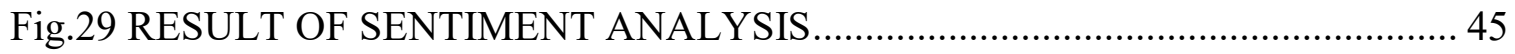

Fig.30 RATING COMPARISON BETWEEN DIFFERENT MODELS ....................... 46 


\section{LIST OF TABLES}

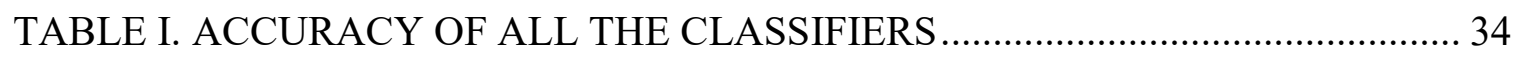

TABLE II. IPHONE 4'S SPECIFICATIONS RATING ............................................ 47 


\section{LIST OF EQUATIONS}

(1) Probability Equation of Naïve Bayes ..................................................... 8

(2) Bayes Theorem Equation for Multiple Variable...................................... 9

(3) Equation in English Form (Naïve Bayes) ….......................................... 9

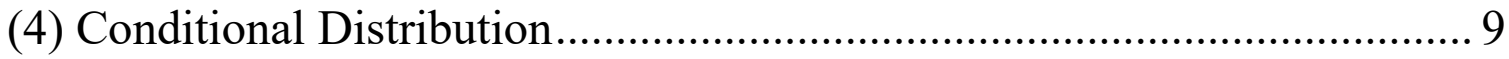

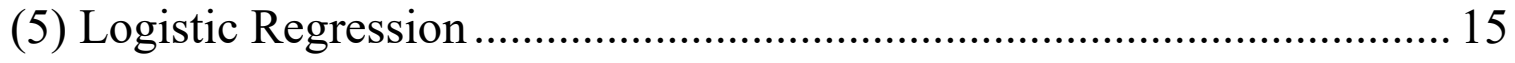

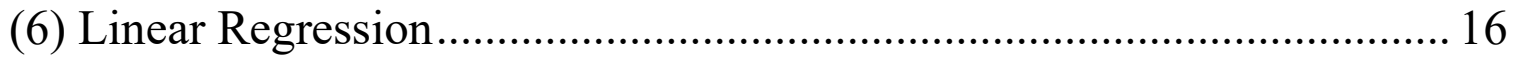

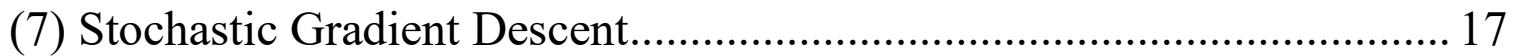

(8) Independently Tree Prediction ............................................................ 18

(9) Conditional Probability for Naïve Bayes ................................................ 31

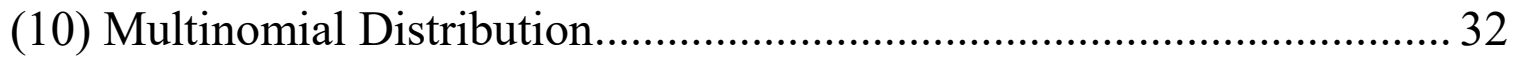

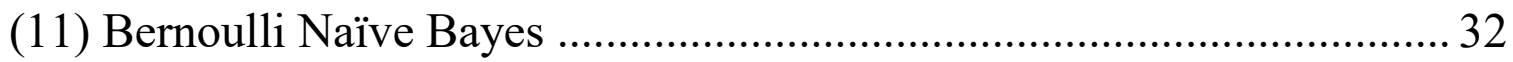

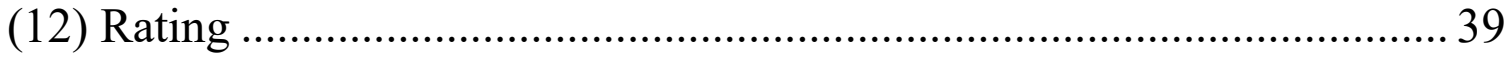

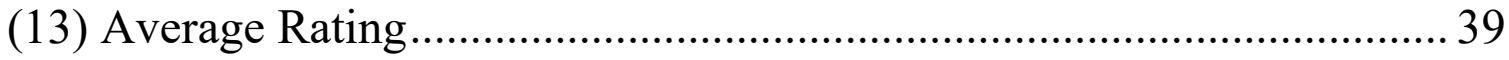




\section{CHAPTER 1 \\ INRODUCTION}

\subsection{OVERVIEW}

Mobile phones have been one of the most essential components for us during the last decade. From Brick Phone to iPhone, technological advancement in mobile phones has taken over our lives. In present, mobile phones have different features according to customer's choice and need. Among thousands of companies, offering their technology to the customers, choosing the appropriate phone for an individual has become an issue. Thus the decision making process becomes more challenging. In this research, we will build a model which generates rating of particular features and overall features, based on public reviews of different features of a mobile phone. The ratings will let customers compare between their needs and public satisfaction and thus choose the suitable mobile phone.

\subsection{MOTIVATION}

There are many websites, Youtube channels and also individuals on Social Media who reviews mobile phones. Professional reviewers are appointed by different companies to act as a marketing strategy for the company. Very few reviewers are authentic and independent of their opinions. Such reviewing criteria benefits the mobile company to convince the customer about their product. This has major drawbacks from the point of view of a customer. Firstly, Professional reviewers, who are appointed by different companies will describe their reviews partially. If there is any lacking in the software or hardware part of the product such as, power consumption, battery lifetime, operating systems operation time, these will be skipped for the sake of the company's marketing strategy, which is not helpful for a customer. Secondly, a mobile phone's technical features, such as Chipset, GPU, 
$\mathrm{CPU}, \mathrm{OS}$ 's contribution to a mobile phone system is not understandable to an average user rather than normal features, such as Camera, Display, Memory. Thus a potential buyer cannot get suitable information that he desires about a mobile phone from the reviewers. Thirdly, reviewers point of explanation of a device do not match the expectation of the customer. A customer's prime concern may about camera while a reviewer will be emphasizing on the technical features. This indicates an absence of a platform where customers can identify the rating of their desired features. Then most importantly, a professional review never reflects the actual state of a device after it is being used for a certain period of time. Generally, reviewers do not use the mobile phones for a period of time and so their reviews do not always contemplate the actual experience of that particular device. Therefore, customers opinion about a mobile phone and its features are more reliable and authentic. This is why comparing ratings of public opinions about a mobile phone to buy one is better rather than relying on professional reviews.

\subsection{CONTRIBUTION SUMMARY}

As public opinion is a deciding factor in many cases, numbers of researches have been done on analyzing the sentiment of public reviews. Very few researches on sentiment analysis has been done in the field of mobile phones which includes mobile application review, caller data analysis and so on. Among these few, none of the researches focused on generating rating based on public reviews and assisting potential customers to buy an appropriate phone according to their needs. The rating of different features and overall mobile phone is a reflection of the user of that device which will advise a customer better than any reviews. In our research, we propose a model that will provide ratings of features and an overall rating of a phone, with which buyers' decision will be relying on public opinion. 


\subsection{THESIS OUTLINE}

The rest of the thesis is organized as follows:

- Chapter 2 includes the necessary background information regarding the proposed approach and the algorithms of sentiment analysis

- Chapter 3 presents the proposed model of the research which includes discussion about datasets, data pre-processing, algorithm implementation, accuracy, sentiment analysis on gathered data.

- Chapter 4 demonstrates the experimental results with graphical representation.

- Chapter 5 concludes the thesis and directs the future research opportunities. 


\section{CHAPTER 2 \\ BACKGROUND STUDIES}

\subsection{LITERATURE REVIEW}

Sentiment analysis is rapidly emerging area of research in natural language processing (NLP). In recent years, this field has gained much interest as uses of internet increased. As the number of consumers' review increases, the need for analyzing these reviews automatically is also increasing which can help today's business to significantly to improve. As a result, not only researchers but also entrepreneurs are becoming interested about sentiment analysis [1]. Sentiment analysis is a machine learning method which can be used to classify and analyze peoples' sentiments, emotions, opinion etc. Review of online product which is expressed in terms of text, star rating, thumbs up and down can be analyzed using sentiment analysis which can significantly help to improve certain products or services. With the aid of negative prefixes, words like adjectives and adverbs are able to carry opposite sentiment. So, in this research, authors used negation phrase identification algorithm to find such words and analyzed the reviews [2]. In some researches, twitter data is used for sentiment analysis. Similarly, in this research, the authors have classified twitter data for US airline service analysis where firstly the tweets are pre-processed to obtain a vector using deep learning concept (Doc2vec) for phrase-level analysis. And then using 7 different classification technique, the analysis is carried out. Based on the accuracy of the result, comparison among different classification technique are given in this research paper [3]. Opinions can also be extracted based on the product's features using several techniques. In this research paper, the authors suggested a system that automatically expand opinion lexicon and based on the dependency relations, the product's features are extracted. 
And to find this dependency relations between features and opinions, StrandfordCoreNLP dependency parser is used and then an algorithm based on double propagation is used to extract feature and opinions. With the aid of Vader lexicon, polarities of extracted opinions are defined [4]. The author Xing Fang and Justin Zhan in their research work describes that when sentiment sentences are extracted, it contains at least one positive or negative word. Tokenization is done to separate the sentences into words and Parts-of-speech (POS) tagging is done. From the original dataset, the tokens and their scores are extracted which are known as features. So, to classify these features, they transformed into vectors [5]. Furthermore, in another paper, the authors used unsupervised learning on large twitter corpora to obtain a word embeddings which uses latent contextual semantic relationships and co-occurrence statistical characteristics between word in tweets. Together with n-grams features and word sentiment polarity score features, these word embeddings form a sentiment feature set of tweets and this feature set is combined into a deep convolution neural network for training and predicting sentiment classification labels. This model shows a significant amount of accuracy in their result [6]. However, authors Roshna Rafeek and R. Remya, in their research work stated that context dependent opinion words are challenging for sentiment analysis because their polarity changes according to the context in which they are used. So, to overcome this challenge, they have proposed a system that uses (aspect, opinion) pairs and logistic regression (LR) model. In this model, to acquire (aspect, opinion) pairs, syntactic rules are used and for classifying the sentiment words into positive class and negative class, LR model is used [7]. In this research paper, the authors proposed an approach for sentiment analysis using Python and its library NLTK (Natural Language Toolkit). Python, which is simple yet powerful, high level, interpreted and dynamic programming language, is very useful for processing natural language using its own library NLTK that helps to build programs and 
classify data. Data can be trained and different numerous classifiers can be tested using NLTK. Additionally, using NLTK, results and patterns can be graphically represented [8]. In another research, the authors described about different supervised machine learning approaches for sentiment analysis. Various algorithms are used and their advantages and disadvantages are stated. There are two broad categories which are Lexicon based approach and machine learning approach. In their research work, they have used three algorithms. These algorithms are naïve bayes, support vector machine and maximum entropy. According to their result, naïve bayes algorithm provided better accuracy than the other two [9].

\subsection{NLTK}

Natural Language Toolkit (NLTK) is a Python-based programming toolkit that works with human language data. This platform provides easy-to-use interfaces to over 50 corpora and lexical resources such as WordNet, along with a suite of text processing libraries for classification, tokenization, stemming, tagging, parsing, and semantic reasoning, wrappers for industrial-strength NLP libraries and an active discussion forum. It was initially released in 2001 and was developed by Steven Bird and Edward Loper in conjunction with a computational linguistics course at the Department of Computer and Information Science at the University of Pennsylvania. It was designed with three pedagogical applications in mind: assignments, demonstration and projects. NLTK supports assignments of varying difficulty and scope. It's interactive graphical demonstration have proven to be very useful for students learning NLP concepts. Additionally, it also provides students with a flexible framework for advanced project. NLTK is implemented as a large collection of minimally interdependent modules, organized into shallow hierarchy. Basic data types which are used throughout the toolkit is defined by a set of core moguls and 
remaining modules are task modules, each devoted to an individual natural language processing task.

\subsection{SUPERVISED LEARNING}

Supervised learning is the machine learning task of learning a function that maps an input to an output based on example input-output pairs. It is simply a formalization of the idea of learning from example where learner, a computer program, is provided with two sets of data, a training set and a test set. The learner "learn" from a set of labeled examples in the training set so that it can identify unlabeled examples in the test set with the highest possible accuracy. The goal of the learner is to develop a rule, a program, or a procedure that classifies new example in the test set by analyzing examples it has been given that already have a class label. In supervised learning, the training set consists of $\mathrm{n}$ ordered pairs $\left(\mathrm{x}_{1}, \mathrm{y}_{1}\right),\left(\mathrm{x}_{1}, \mathrm{y}_{2}\right), \ldots,\left(\mathrm{x}_{\mathrm{n}}, \mathrm{y}_{\mathrm{n}}\right)$, where each $x_{i}$ is some measurement or set of measurements of a single example data point, and $\mathrm{y}_{\mathrm{i}}$ is the label for that data point. For example, an $\mathrm{x}_{\mathrm{i}}$ might be a group (sometimes called a vector) of five measurements for a patient in a hospital including height, weight, temperature, blood sugar level, and blood pressure. The corresponding $\mathrm{y}_{\mathrm{i}}$ might be a classification of the patient as "healthy" or "not healthy". The test data in supervised learning is another set of $\mathrm{m}$ measurement without labels: $\left(\mathrm{x}_{\mathrm{n}+1}, \mathrm{x}_{\mathrm{n}+2}, \ldots, \mathrm{x}_{\mathrm{n}+\mathrm{m}}\right)$. As described above, the goal is to make educated guesses about the labels for the test set (such as "healthy" or "not healthy") by drawing inferences from the training set. 


\subsection{ALGORITHMS}

To implement our research on supervised machine learning for sentiment analysis, we need to use different algorithms. We use Naïve Bayes classifiers, Support Vector Machine classifiers, Logistic regression, linear regression, Stochastic gradient descent classifier and Random forest.

\subsubsection{NAÏVE BAYES}

A Naive Bayes classifier is an algorithm that uses Bayes' theorem to classify data. Naive Bayes classifiers assume strong, or naive, independence between attributes of data points. The classifier uses probability theory to classify objects. The main key insight of Bayes' theorem is that the probability of an event can be adjusted as new data is introduced. A Naïve Bayes classifier is called naïve because its assumes that all attributes of a data point under consideration are independent of each other. A Naïve Bayes model is easy to build and it has no complicated iterative parameters estimation which makes it particularly useful for very large datasets [10]. Though it is simple model but it often does surprisingly well and it is widely used because it outperforms more sophisticated classification methods. As mentioned earlier Bayes theorem provides a way to calculate the posterior probability $P(c \mid x)$, from $P(c), P(x)$, and $P(x \mid c)$. Naive Bayes classifier assume that the effect of the value of a predictor $(x)$ on a given class $(c)$ is independent of the values of predictors. The mathematical representation of equation is given below

$$
p(c \mid x)=\frac{p(x \mid C) p(C)}{p(X)}
$$

Here,

$P(c \mid x)$ is the posterior probability of target class (c) given predictor (x). 
$P(c)$ is the prior probability of class.

$P(x \mid c)$ is the probability of predictor given class.

$P(x)$ is the prior probability of predictor class.

Naive Bayes classifiers can handle an arbitrary number of independent variables whether continuous or categorical. Abstractly this probability model for a classifier is a conditional model. Over an independent variable $\mathrm{X}$ with a small number of outcomes or classes on several feature variable $\boldsymbol{y}_{\boldsymbol{1}}$ through $\boldsymbol{y}_{\boldsymbol{n}}$. When the number of features $\mathbf{n}$ is large we therefore reformulate the formula to make it more tractable.

$$
\mathrm{P}\left(\mathrm{x} \mid \mathrm{y}_{1} \ldots \ldots \ldots \mathrm{y}_{\mathrm{n}}\right)
$$

Using Bayes theorem, we can write,

$$
\mathrm{P}\left(\mathrm{x} \mid \mathrm{y}_{1} \ldots \ldots \ldots \mathrm{y}_{\mathrm{n}}\right)=\frac{p(X) p(Y 1 \ldots \ldots . . \mathrm{Yn} \mid X)}{p(Y 1 \ldots \ldots . . \mathrm{Yn})}
$$

In plain English above equation will be written as

$$
\text { Posterior }=\frac{\text { Prior } * \text { likelihood }}{\text { evidence }}
$$

This means that under the above independence assumptions, the conditional distribution over the class variable $\mathrm{C}$ can be expressed like this:

$$
\mathrm{P}\left(\mathrm{x} \mid \mathrm{y}_{1} \ldots \ldots \ldots \mathrm{y}_{\mathrm{n}}\right)=\frac{1}{z} \mathrm{p}(\mathrm{c}) \prod_{i=1}^{n} p(Y \mathrm{i} \mid \mathrm{X})
$$

Where $\mathrm{Z}$ is factor dependent of $\mathrm{Y}_{1}$ though $\mathrm{Y}_{\mathrm{n}}$.

In this research paper Scikit learn (python library) has been used. In Scikit learn there are three different types of Naïve Bayes model under the library. 


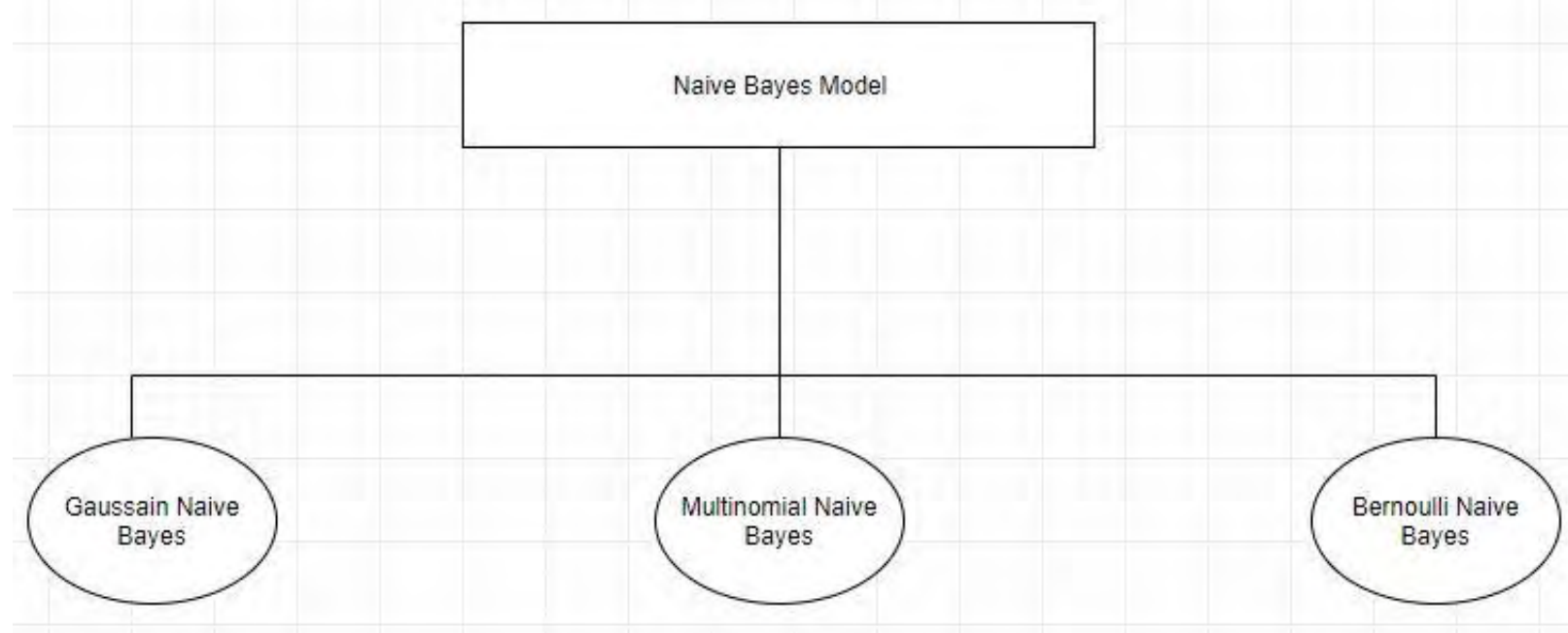

Fig. 1. Types of Naïve Bayes classification

In our research Paper We use Multinomial Naïve Bayes and Bernoulli Naïve Bayes algorithms using Scikit learn library. Multinomial Naïve Bayes estimates the conditional probability of a particular word given a class as the relative frequency of term in data belongings to class. Bernoulli generates Boolean value about each term of the specific data.

\subsubsection{SUPPORT VECTOR MACHINE}

Support Vector Machine (SVM) is a supervised machine learning algorithm which can be used for both classification or regression challenges. However, it is mostly used for test classification. In the algorithm each data will be plot as a point in ndimensional space with the value of each feature. $n$ is the number of features. Then classification is done to find the hyper-plane to differentiate two classes very well. Normally this model draws lines to separate the groups according to patterns [11]. 


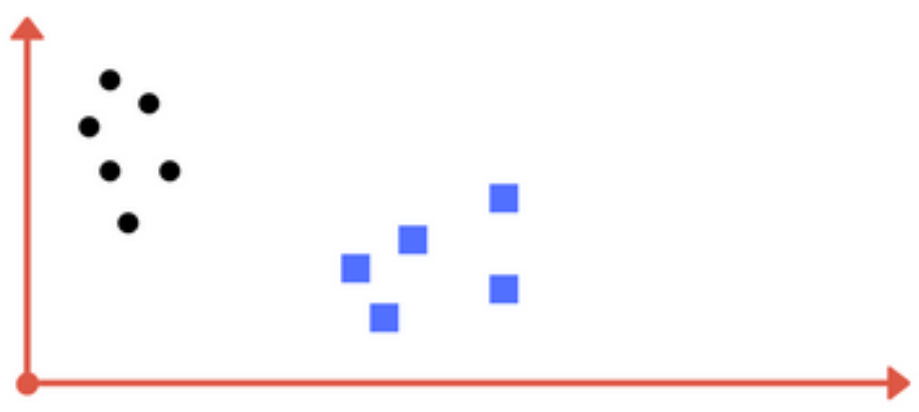

Fig. 2. SVM Classifications of two classes

Here in figure 2 there are two different class in the graph. Support Vector Machine will find a hyper-plane to separate two different classes as figure 3 .

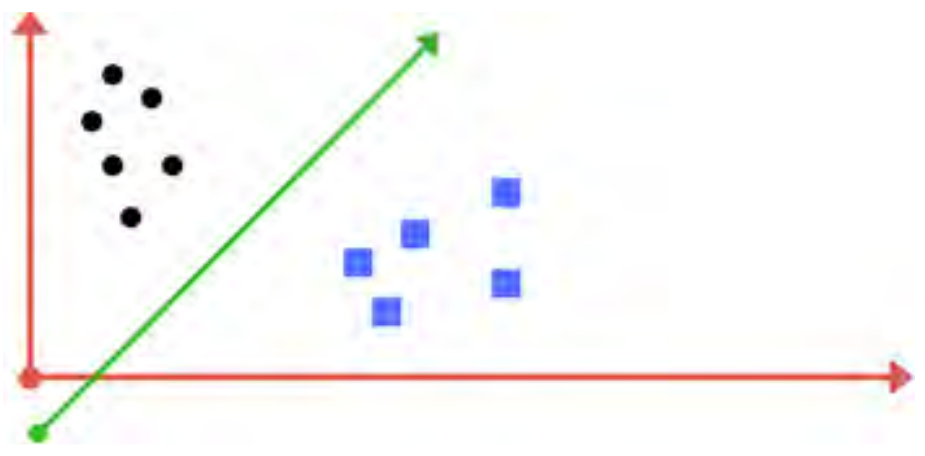

Fig. 3. SVM classification using hyper-line

The distance between the hyperplane and the nearest data point from either set is known as the margin. The goal is to choose a hyperplane with the greatest possible margin between the hyperplane and any point within the training set, giving a greater chance of new data being classified correctly. 


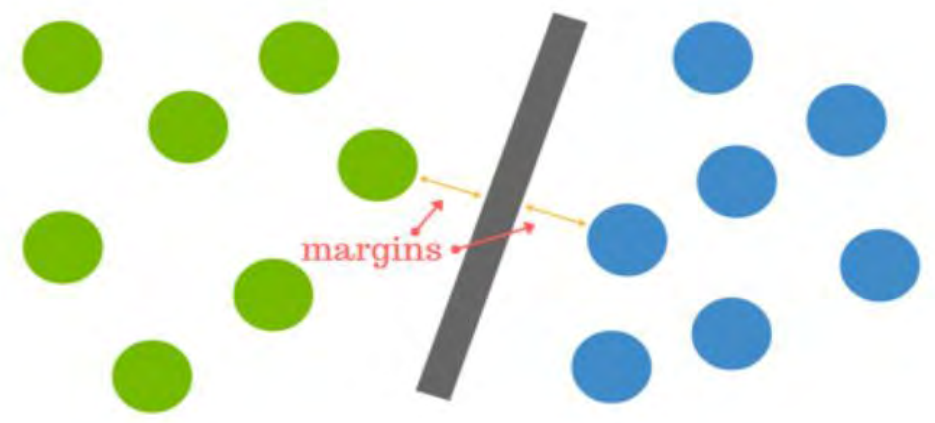

Fig. 4. Best hyper-plane

The above is a classic example of a linear classifier. A classifier that separates a set of objects into their respective groups (GREEN and BLUE in this case) with a line. Most classification tasks, however, are not that simple, and often more complex structures are needed in order to make an optimal separation. In real world data is rarely as clean as above. Datasets will be mixed as figure 5 shown below

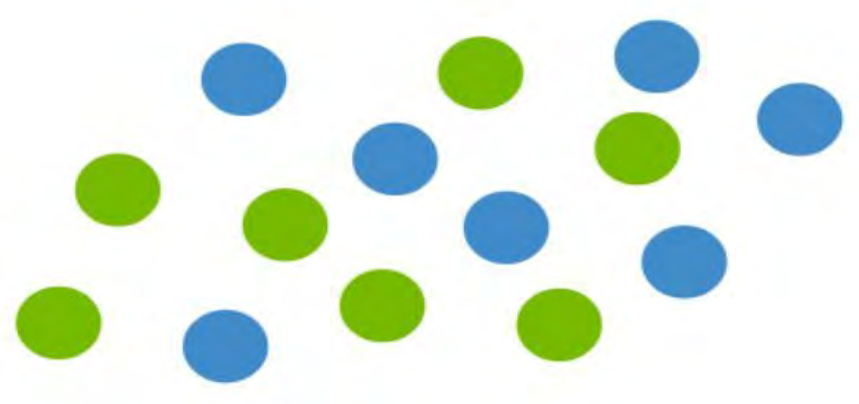

Fig. 5. SVM classifications with jumbled data

To classify above jumbled dataset, it is necessary to move away from 2D view of the data to $3 \mathrm{D}$ view. Normally for jumbled datasets it is most important to represent all the data to higher dimension. This is called kernelling .AS we consider our dataset in higher dimension our hyper-plane will not be a line this time, it will be shown as figure 6 below 

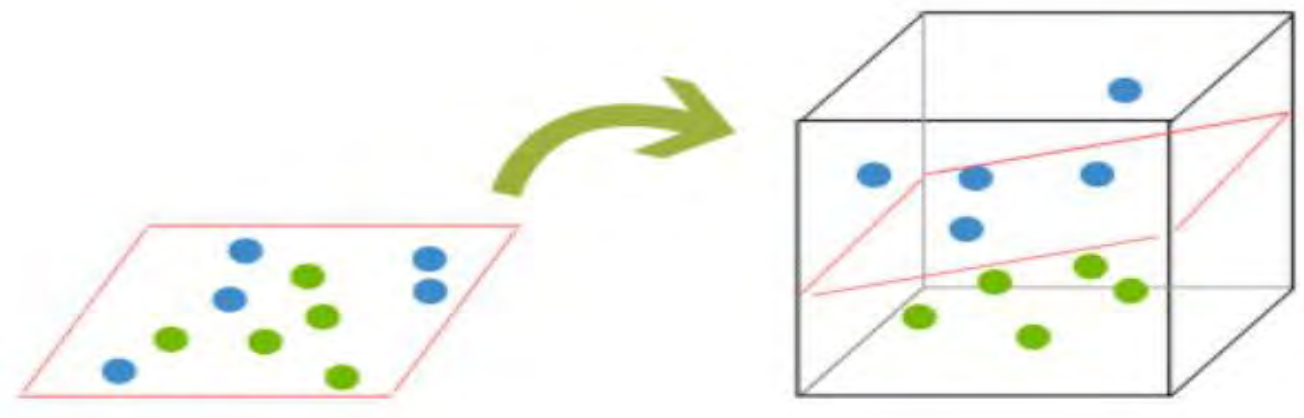

Fig. 6. SVM classification in 3D dimension

To construct a hyper-plane line SVM employs an iterative training algorithm, which is used to minimize an error function. According to the form of the error function, SVM models can be classified into four distinct types that is shown in Figure 7.

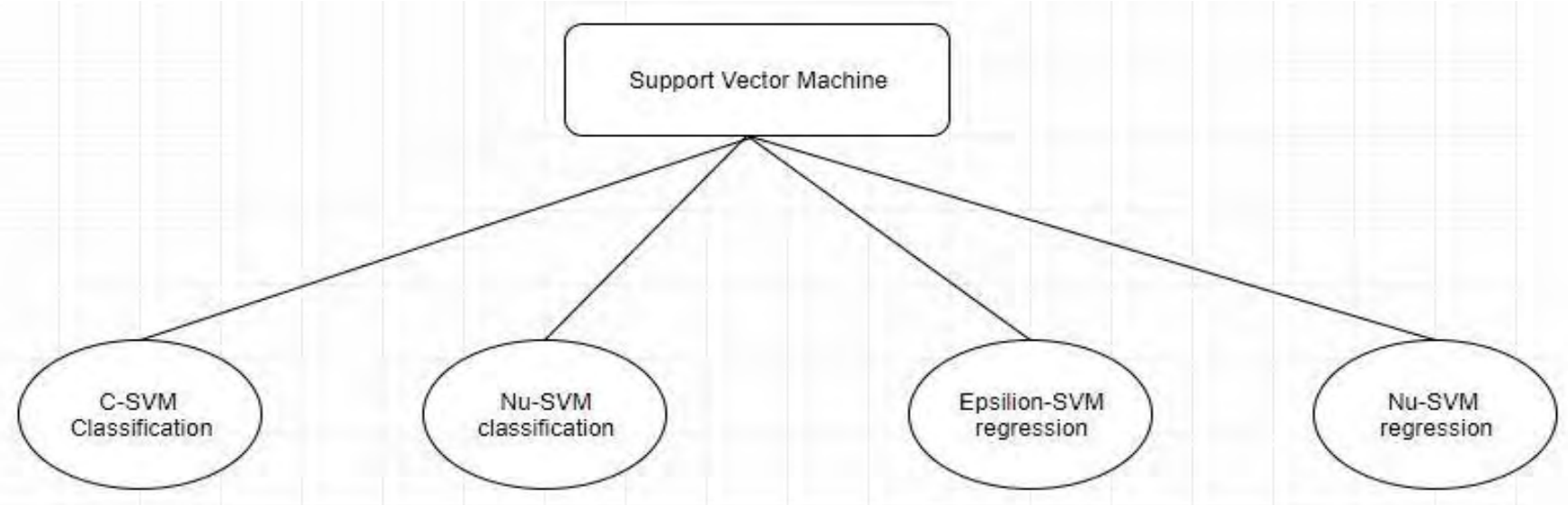

Fig. 7. Types of SVM classification

In our research we used Scikit learn library to implement the support vector machine algorithm. In Scikit lean library there are different implementation of the support 
vector machine algorithm such as NuSVC, Linear SVC. In the research, NuSVC and Linear SVC are used for sentiment analysis.

\subsubsection{REGRESSION}

Regression analysis is the method of using observations (data records) to quantify the relationship between a target variable (a field in the record set), also referred to as a dependent variable, and a set of independent variables. Regression analysis is an important tool for modelling and analyzing data. There are various kind of regression techniques available to make predictions. These techniques are mostly driven by three parameters. Parameters are given below in figure 8

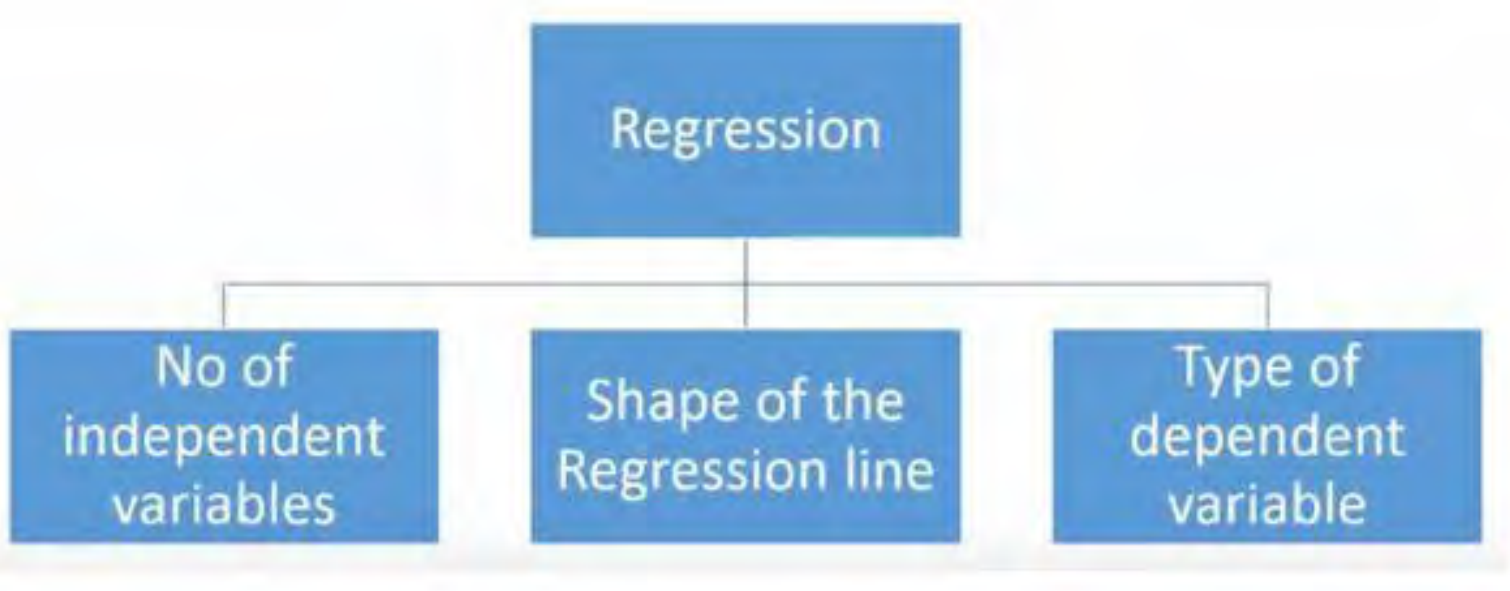

Fig. 8. Parameters of regression model

\subsubsection{LOGISTIC REGRESSION}

Logistic regression is the appropriate regression analysis to conduct when the dependent variable is binary. Like all regression analyses, the logistic regression is a predictive analysis. Logistic regression is used to describe data and to explain the 
relationship between one dependent binary variable and one or more nominal, ordinal, interval or ratio-level independent variables. This model aims to measure the relationship between categorical dependent variable and one or more independent variables by plotting the dependent variables' probability scores [12]. As probability scores is a ratio, what will be actually modeled is the logarithm of the

probability. The mathematical representation of equation is given below as figure 9 .

$$
\log \left(\frac{\pi}{1-\pi}\right)=\beta_{0}+\beta_{1} x_{1}+\beta_{2} x_{2}+\ldots \beta_{m} x_{m}
$$

Here,

$\Pi$ indicates the probability of an event.

$\beta_{i}$ are the regression coefficients.

$x_{i}$ explanatory variables.

In the research paper this model is used to determine the output or result when there are one or more than one independent variables. The output value can be in form of 0 or 1 in binary form.

\subsubsection{LINEAR REGRESSION}

Linear regression is one of the most widely known modeling technique. Linear regression is usually among the first to few methods of regression. It is a technique in which the dependent variable is continuous in nature, independent variables can be continuous or discrete and nature of regression line is linear. Linear Regression establishes a relationship between dependent variable(Y) and one or more 
independent variables( $\mathrm{X})$ using a best fit straight line. It is represented by an equation, it is given below

$$
\mathrm{Y}=\mathrm{a}+\mathrm{b} * \mathrm{X}+\mathrm{e}
$$

Here,

a is intercept.

$\mathrm{b}$ is slope of the line.

$\mathrm{e}$ is error term.

In our research it is used to predict the value of target variable based on given predictor variables [12].

\subsubsection{STOCHASTIC GRADIENT DESCENT}

Stochastic gradient descent (SGD) is an iterative optimization algorithm that can be applied to functions that are a linear combination of differentiable functions. These types of functions often arise when the full objective function is a linear combination of objective functions at each data point. This involves knowing the form of the cost along with the derivative so that from given point that is already a known gradient can move in the direction. Stochastic gradient descent approximates the full gradient by using the gradient at each of the functions in the linear combination. Gradient descent might be slow to implement for large number of datasets. This is where Stochastic gradient descent is used. Stochastic gradient descent is a machine learning algorithm that is capable of making thee classifier learn even if it's based on nondifferentiable loss function [14]. It evaluates and updates the coefficients every iteration to minimize the error of a model on the training data. Each instance of 
training data is shown to the model at a time. After calculating the error, the model is updated to reduce the error for the next iteration. This procedure is used to find the set of coefficients in a model that result in the smallest error on the training data. Coefficients are updated using the equation below

$$
\mathrm{b}=\mathrm{b}-\text { learning_rate } * \text { error } * \mathrm{x}
$$

Here,

$\mathrm{b}$ is the coefficient being optimized.

Learning rate is a hyper-parameter that controls the weights of our network with respect the loss gradient.

Error is the prediction error for the model on the training data attributed to the coefficients.

$\mathrm{x}$ is the input value.

\subsubsection{RANDOM FOREST}

Random forests, also known as random decision forests, are a popular ensemble method that can be used to build predictive models for both classification and regression problems. It is a flexible, easy to use machine learning algorithm that produces, even without hyper-parameter tuning, a great result most of the time. It is also one of the most used algorithms, because it's simplicity and the fact that it can be used for both classification and regression tasks [13]. Random Forest is a supervised learning algorithm. As its name, it creates a forest and makes it somehow random. The 'forest' it builds, is an ensemble of Decision Trees, most of the time trained with the "bagging" method. The general idea of the bagging method is that a combination of learning models increases the overall result. Simply random forests 
build multiple decision tress and merge them to get more accurate and stable predictions. There are three main choices to be made when constructing a random tree. These are (1) the method for splitting the leafs, (2) the type of predictor to use in each leaf, and (3) the method for injecting randomness into the trees. Each tree is grown as follows:

1. If the number of cases in the training set is $\mathrm{N}$, sample $\mathrm{N}$ cases at random - but with replacement, from the original data. This sample will be the training set for growing the tree.

2. If there are $M$ input variables, a number $m<<M$ is specified such that at each node, $\mathrm{m}$ variables are selected at random out of the $\mathrm{M}$ and the best split on these $\mathrm{m}$ is used to split the node. The value of $\mathrm{m}$ is held constant during the forest growing.

3. Each tree is grown to the largest extent possible. There is no pruning.

The correlation between any two trees in the forest. Increasing the correlation increases the forest error rate. The strength of each individual tree in the forest. A tree with a low error rate is a strong classifier. Increasing the strength of the individual trees decreases the forest error rate. Once the forest has been trained it can be used to make predictions for new unlabeled data points. To make a prediction for a query point $\mathrm{x}$, each tree independently predicts as below equation

$$
f_{n}^{j}(x)=\frac{1}{N^{e}\left(A_{n}(x)\right)} \sum_{\substack{Y_{i} \in A_{n}(x) \\ I_{i}=e}} Y_{i}
$$

Here $\operatorname{An}(\mathrm{x})$ denotes the leaf containing $\mathrm{x}$.

$\mathrm{N}^{\mathrm{e}}(\operatorname{An}(\mathrm{x}))$ denotes the number of estimation points it contains. 
The predictions made by each tree depend only on the estimation points in that tree. However, since points are assigned to the structure and estimation parts independently in each tree, structure points in one tree have the opportunity to contribute to the prediction as estimation points in another tree.

In our research, we use random forest for text classification purpose. In python we use random forest(rf) library to implement the code. 


\section{CHAPTER 3 \\ PROPOSED MODEL}

\subsection{MODEL OVERVIEW}

For textual sentiment analysis, the best algorithm that researchers used in past is Naïve Bayes algorithm. Our model consists of Naïve Bayes as well as some other algorithms. There are nine steps that we have followed in our model.

1. Data collection.

2. Data Splitting into train and test sets.

3. Data pre-processing, that consists of text cleaning or mining.

4. Choosing the most common features from the train data.

5. Training and testing the algorithms with the dataset.

6. Using classifier to choose the best algorithm.

7. Testing the accuracy of the classifier.

8. Applying sentiment analysis on the review data, with individual features to get polarity.

9. Finding the average polarity of each feature along with the average sentiment polarity of a model.

10. Providing ratings of each model of mobile phones analyzing the average polarity.

Figure 9 illustrates the proposed model in block diagram, 


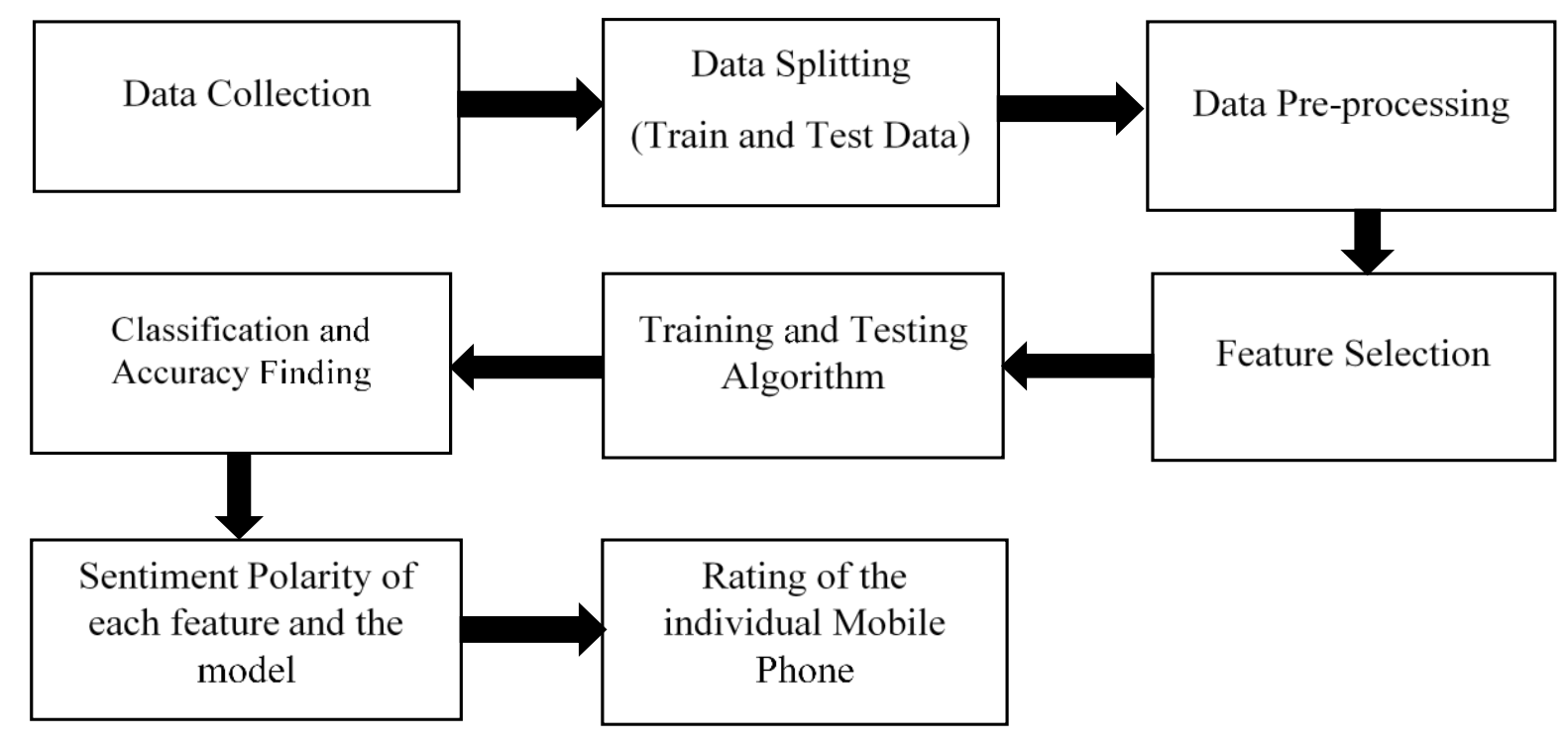

Fig. 9. Block Diagram of the proposed Model

\subsection{DATA COLLECTION}

In the research of sentiment analysis, the most technical and one of the most tough tasks is to find out the proper dataset applicable for the model. As our aim is to find out the proper review and rating of a mobile set we generalized our searching criteria for datasets with proper reviews along with mobile set company or model name. We used the Amazon's unlocked mobile phone review's dataset [1]. There were four hundred thousand data available in the dataset. The dataset contained in total six columns, which were 'product name', 'company name', 'review', 'rating', 'rating votes'. Three of the columns consists of textual data and rest are numerical data. The 'Product Name' column is the model name of the mobile phone whereas the 'Company Name' is the name of the company. The review column consists of reviews provided by users which are sometimes in multiple and sometimes in one sentence. Rating column is the average rating of each review given by users at Amazon. 


\subsection{DATA SPLITTING}

Data splitting is the process of splitting the dataset into training and testing data. This process is very useful for any machine learning process as the main idea of machine learning depends on training and testing data and finding the accuracy of the machine given result. Firstly, we had to reduce our data to two thousand from four hundred thousand as the machine's processing power couldn't handle this amount of big data. In our research, we divided our dataset because we will train our algorithms on the test dataset where the review rating is already given. Here the algorithms will be trained using the review and the rating column. We set the rating 4 and 5 as good rating and 1 and 2 as bad rating. The algorithms will be trained with following information and we will apply the trained algorithm to our test set and measure the accuracy of the machine.

The datasets are usually divided into a 80:20 ratio. However, for our model the dataset was split into $70: 30$ ratio. Thus, the $70 \%$ of the selected data is chosen as training set and the rest $30 \%$ is test set. There is many built in python toolkits for splitting data such as, 'panda', 'Keras', 'Scikit learn' etc. although we used Scikit learn for the machine learning approaches in this research because of its built-in libraries.

\subsection{DATA PRE-PROCESSING}

Data preprocessing is the process of cleaning the data to an extent where the machine learning algorithm will understand the content of the data. Overall the data preprocessing deals with finding the inaccurate, irrelevant and incomplete data and removing or those data to minimizing the text. Pre-processing is very important in 
terms of textual data. This pre-processing task is done using NLTK (Natural Language Tool Kit) which is an open source tool for textual data mining. There are several steps of text pre-processing, which are

\section{Tokenization}

\section{Normalization}

3. Noise removal

The block diagram of text pre-processing is shown in Figure 10

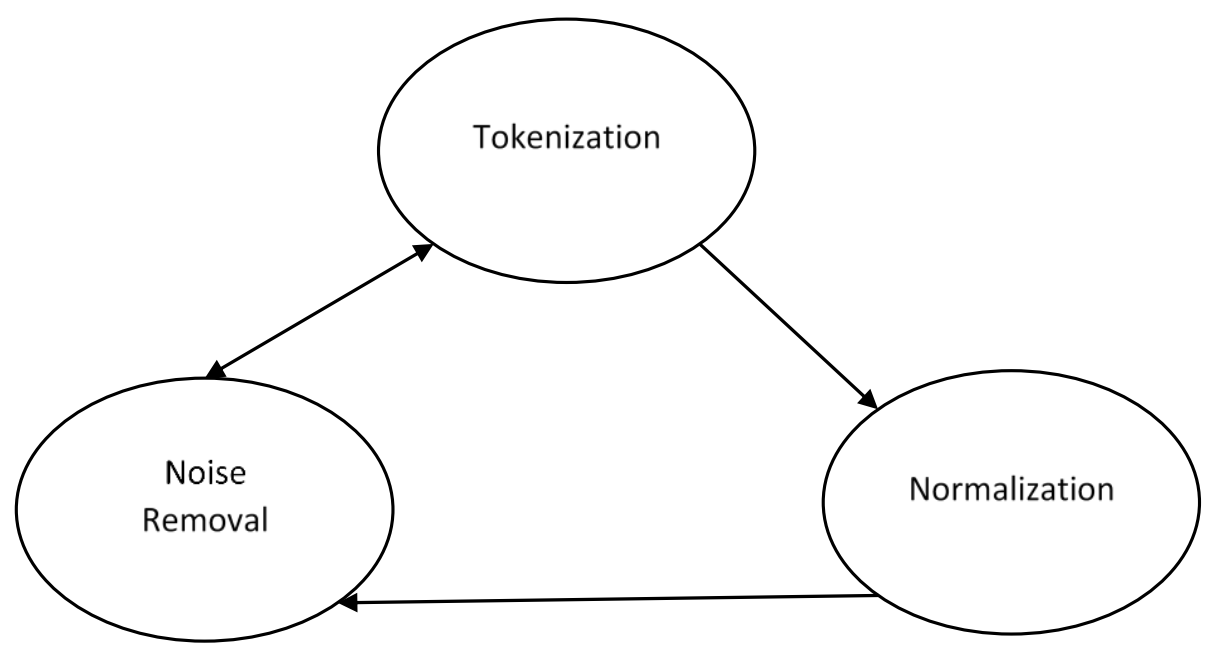

Fig 10. Block diagram of text pre-processing

1. Tokenization:

Tokenization is a step which splits longer strings of text into smaller pieces, or tokens. Larger chunks of text can be tokenized into sentences and sentences can be tokenized into words, etc. Further processing is generally performed after a piece of text has been appropriately tokenized. Tokenization is also referred to as text segmentation or lexical analysis. Sometimes segmentation is used to refer to the breakdown of a large chunk of text into pieces larger than words (e.g. paragraphs or sentences), 
while tokenization is reserved for the breakdown process which results exclusively in words. As an example, one of the reviews from the dataset was, "I feel so LUCKY to have found this use (phone to us \& not used hard at all), phone on line from someone who has upgraded and sold this one to me. My Son liked his old one and and that finally fell apart after 2.5+ years and didn't want an upgrade!! Thank you Seller, we really appreciate it \& your honesty re: said used phone.I recommend this seller very highly \& would but from them again!!". The review is not clean and it contains some words which are not understandable. In Figure 11 the word tokenized version of the review is given which separates all the words in the sentence.

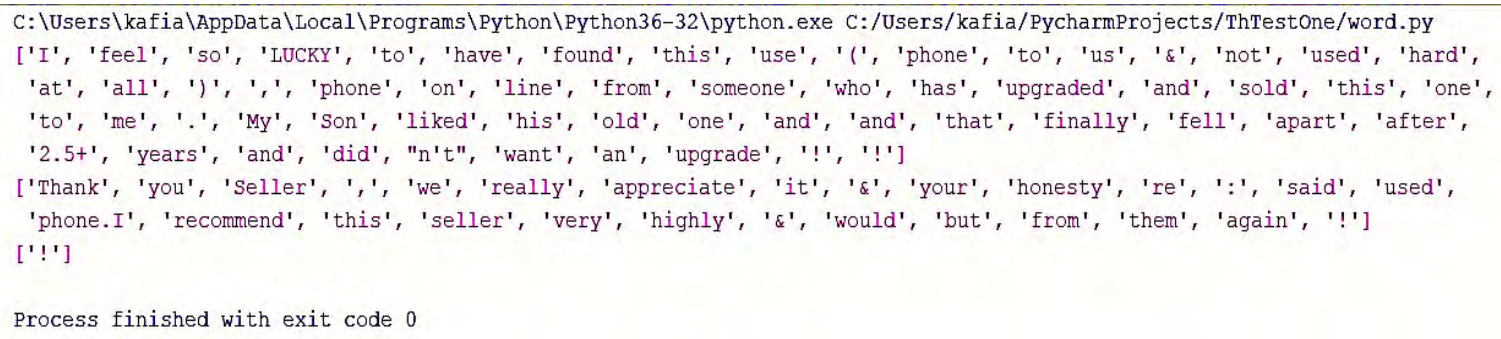

Fig. 11. Tokenized version of the review

2. Normalization:

Before further processing, text needs to be normalized. Normalization generally refers to a series of related tasks meant to put all text on a level playing field: converting all text to the same case, upper or lower case, removing punctuation, converting numbers to their word equivalents, and so on. Normalization puts all words on equal footing and allows processing to proceed uniformly. 
Normalizing text can mean performing a number of tasks, but for our model we approached normalization in three distinct steps: (1) stemming, (2) lemmatization (3) Other process

i. Stemming: Stemming is the process of eliminating affixes (suffixed, prefixes, infixes, circumfixes) from a word in order to obtain a word stem. For an example, the word running is stemmed to run, going stemmed to go. Figure 12 shows an example of applying stemming to a sentence.

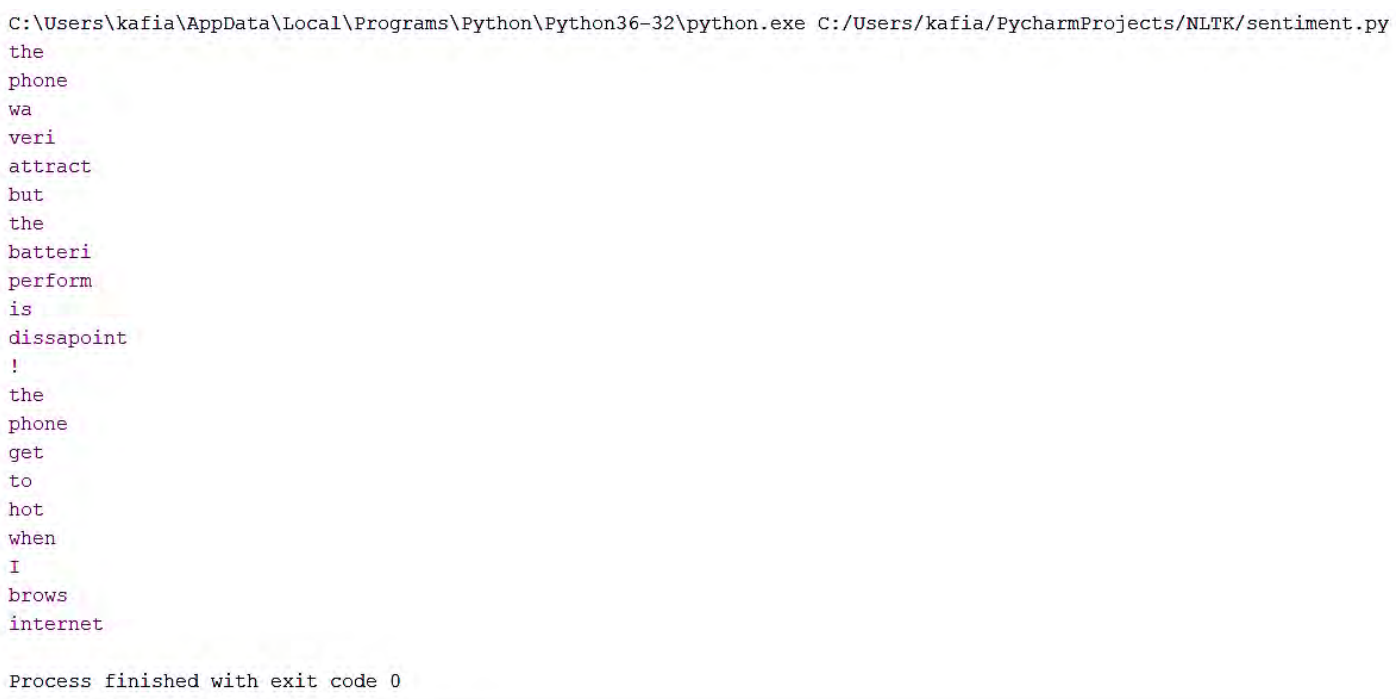

Fig 12. Result after applying stemming

ii. Lemmatization: Lemmatization is related to stemming, differing in that lemmatization is able to capture canonical forms based on a word's lemma. For example, stemming the word "cacti", "geese", "rocks" would fail to return its citation form (another word for lemma); however, lemmatization would result, "cactus", "goose", "rock". Figure 13 shows an example of applying lemmatization to a sentence. 


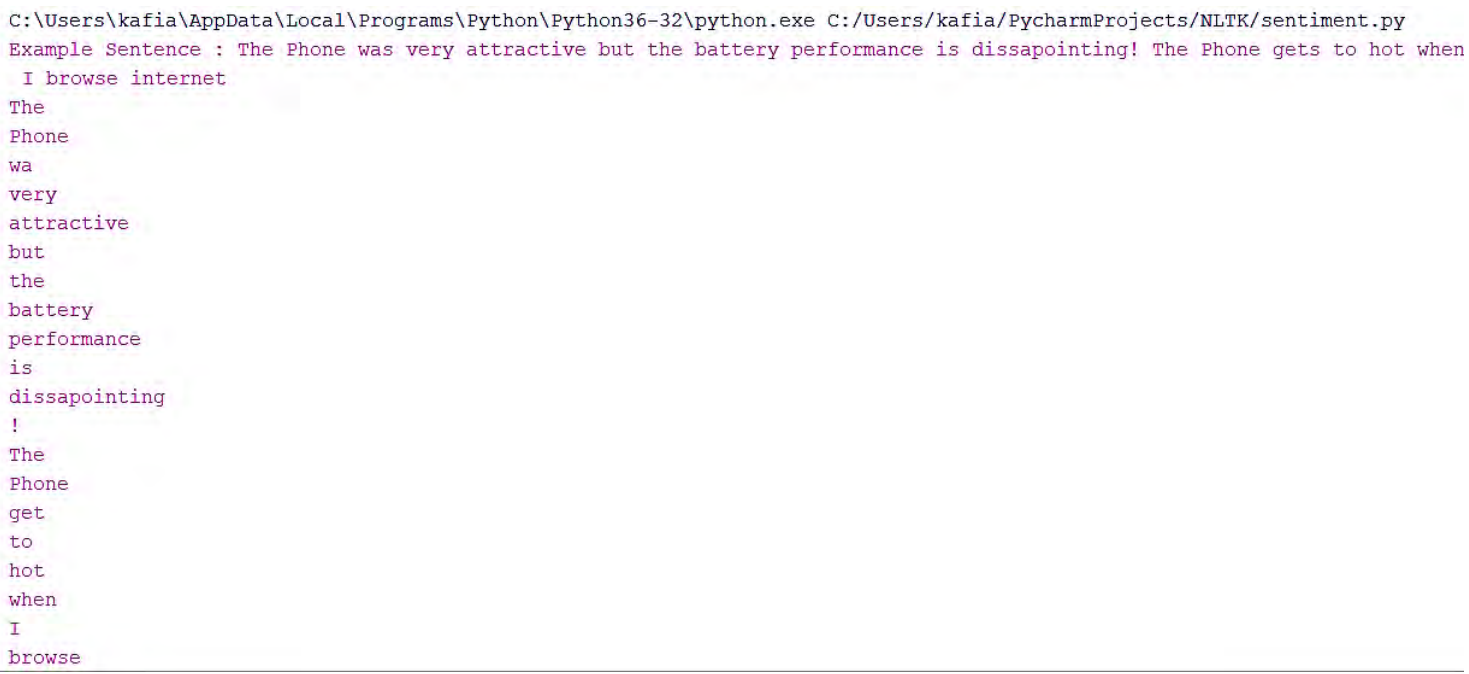

Fig. 13. Result of applying lemmatization

iii. The other processes include setting all the characters to lower or upper case, removing numbers, removing punctuation, removing stop words. Stop words are those words which are filtered out before further processing of text, since these words contribute little to overall meaning, given that they are generally the most common words in a language. For instance, "the," "and," and "a," while all required words in a particular passage, don't generally contribute greatly to one's understanding of content. For an example, "The Phone was very attractive but the battery performance really dissapoints! The Phone gets to hot when I browse internet" is a review sentence without removing stop words. Figure 14 shows the sentence after removing stop words. 
C: \Users \kafia\AppData\Local\Programs\Python\Python36-32\python.exe C:/Users/kafia/PycharmProjects/ThTestone/word.py ['The', 'Phone', 'was', 'very', 'attractive', 'but', 'the', 'battery', 'performance', 'really', 'dissapoints', '!',

'The', 'Phone', 'gets', 'to', 'hot', 'when', 'I', 'browse', 'internet']

['The', 'Phone', 'attractive', 'battery', 'performance', 'really', 'dissapoints', '!', 'The', 'Phone', 'gets', 'hot',

'I', 'browse', 'internet']

Process finished with exit code 0

Fig. 14. Result of removing Stop words

POS tagging is also a part of text pre-processing and used for detecting the parts of speech of the words in a sentence. For selecting the adjective of a sentence, we use this process in sentiment analysis. An adjective can determine the polarity of a sentence or word. Figure 15 shows the result of applying pos tagging to a review sentence.

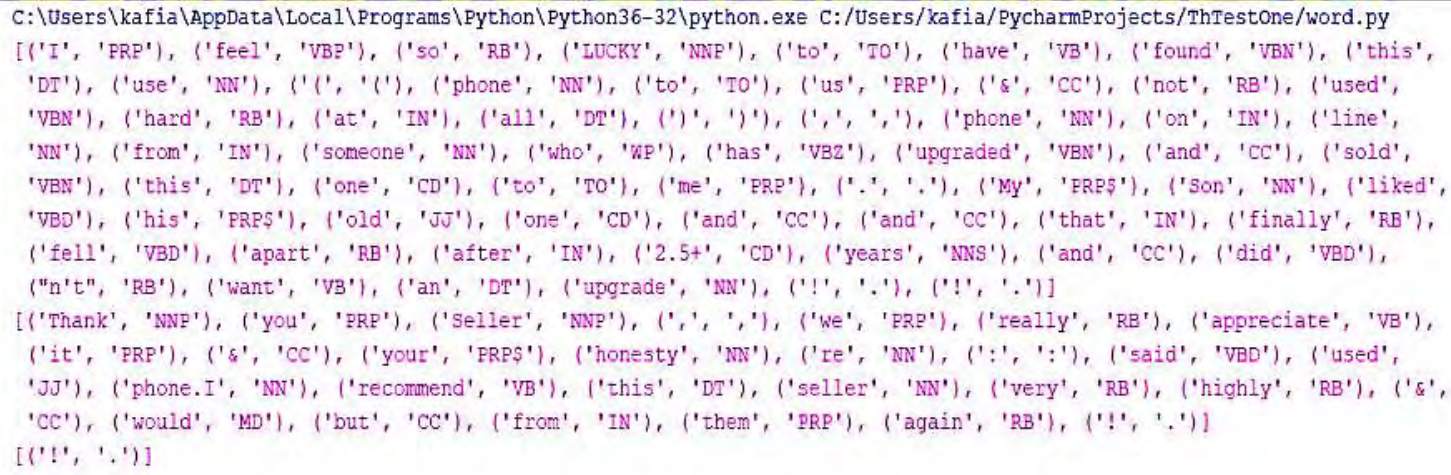

Fig. 15. Result of applying POS tagging

3. Noise Removal: Noise removal continues the substitution tasks of text preprocessing. While the first 2 major steps of our model were generally applicable as-is to nearly any text chunk or project noise removal is a much more task-specific section of the model. Noise removal works as a tool to remove text headers, footers, extracting text from special file formats. 


\subsection{FEATURE OF MOBILE PHONE SELECTION}

Our model concentrates on finding out the most frequently reviewed specification of the mobile phones, so the selection process is a vital process in the working methodology. Mobile phones have multiple features like, Camera, RAM, Storage, Battery, Display, Operating system etc. We first created a list which consists of features of mobile phone. As a single feature has multiple name, we applied NLTK synonyms for the features and stored all the strings in a single array. Next, we search for the most frequent feature in the whole dataset by matching with our array. Figure 16 shows the most common features of our dataset.

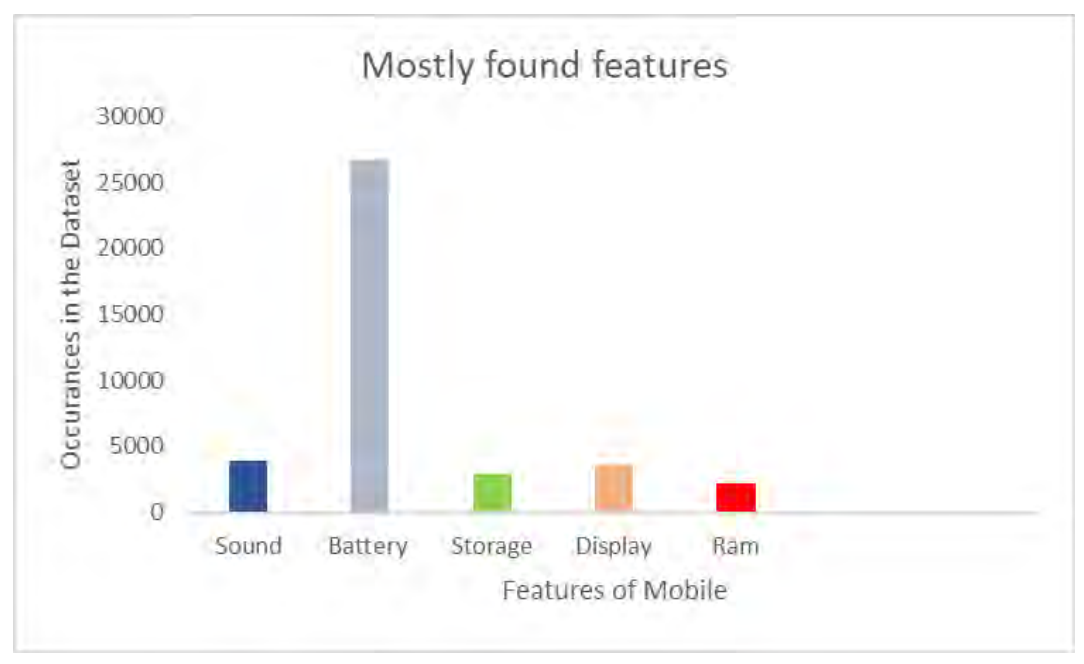

Fig. 16. Most common features of train data

We selected in total 15 common features to do our research. In figure 17 the most common features of our model is shown. These features are mostly noun which defines the specification of any mobile phone. 


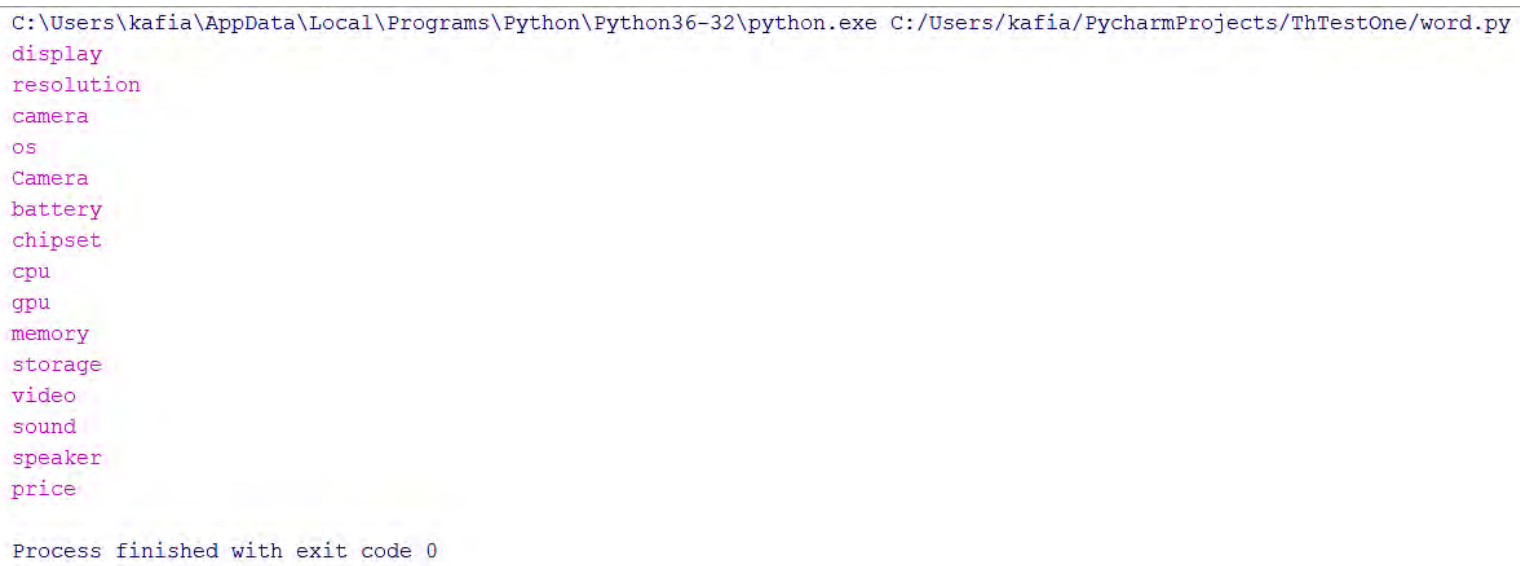

Process finished with exit code 0

Fig 17. Selected 15 features of the model

\subsection{TRAINING AND TESTING ALGORITHMS}

Training algorithm with the test dataset is one of the most important tools for the success of any research. As the algorithms are not suitable enough for any model, the concept of training the dataset and making the algorithm more useful is a core concept in machine learning. To train the dataset and to see which algorithm fits better in our model, multiple algorithms are used. We have to apply the algorithms and compare them to see which of the algorithm suits our model and gives better result. In this research we have choose $70 \%$ of the dataset to be the training data. As the dataset contains mostly textual data, we are going to use Naïve Bayes, Support Vector Machine, Logistic Regression, Logistic Regression, Multinomial Naïve Bayes, Stochastic Gradient Descent algorithms. For machine learning Keras and Tensorflow is widely used by researchers. As mentioned earlier, in our research we are going to use 'Scikit Learn' which is a machine learning library for python programming language. Moreover, for the textual processing we are using NLTK. However, for training the dataset we use the columns, 'review' and 'rating'. The algorithms will be trained with the pre-processed textual review and the numerical ratings. We have to find out the most common adjectives in the dataset which 
determines the polarity of the review. The ratings are within 1 to 5 where we decided 1 and 2 to be 'bad' and 3 and 4 to be 'good'. We have to apply the scikit learn built in data frame for training the both columns as $\mathrm{x}: \mathrm{y}$ where $\mathrm{x}$ would be the review data and $y$ the rating of the review.

After training the algorithms with the trainset we will test the algorithm with the test dataset. As mentioned earlier, $30 \%$ of our dataset is now test data after the split. As we already trained the algorithms with two columns, now we only test the dataset with the review column which will give the output in ratings. So, by measuring the test data and train data we can easily find out the accuracy. However, the accuracy mainly depends on the number of feature sets which consists of only the preprocessed textual data or adjective. The more number of feature set gives more accuracy.

\subsection{CLASSIFICATION AND ACCURACY FINDING}

As earlier mentioned, there is not a single algorithm which fits to a model accurately. Therefore, we have used multiple algorithms for training our dataset. We increased the feature set gradually to see the accuracy of the algorithms and compared them to get the best fit for our model. Classification is the task of choosing the correct class label for a given input. In the basic classification tasks, each input is considered in isolation from all other input, and the set of the labels is defined in advance. Mentioned earlier, we using the built in libraries to do all the classification process. To be specific, we are using the following classifier for our model.

- Naïve Bayes (NB), Multinomial Naïve Bayes (MNB), Bernoulli Naïve Bayes $(\mathrm{BNB})$

- Support Vector Classifier, NuSVC and Linear SVC 
- Logistic Regression and Linear Regression

- Stochastic Gradient Descent (SGD) Classifier

- Random Forest

Naïve Bayes classifier is a simple probabilistic classifier which follows the Bayes theorem with strong independent assumption. This classifier is also said independent feature model. Naïve Bayes classifier assumes the presence of a particular feature of a class which is unrelated to the presence or absence of any other class. Naïve Bayes classifier works very fine in terms of textual data classification and can really be a helpful classifier in terms of real world problems. In our model, we considered feature set which consisted of adjective in which we did not selected the positive and negative words. The probability of a review belongs to a class $C$ is given by the class probability $P(C)$ multiplied by the products of the conditional probability of each word for that class.

$P=P(C) \cdot \prod_{i} P(d i \mid C)=P(C) \cdot \prod_{i}^{n} \frac{\operatorname{count}(d i, C)}{\sum_{i} \operatorname{count}(d i, C)}=P(C) \cdot \prod_{i} \frac{\operatorname{count}(d i, C)}{V c}$

Here, count $(d i, C)$ is the number of occurrences of the word $d i$ in class $C, V c$ is the total number of words in class $\mathrm{C}$ and $\mathrm{n}$ is the number of words in the review we are classifying.

Multinomial Naïve Bayes is a special Naïve Bayes classifier that is designed for classifying text documents. It estimates the conditional probability of a particular word given a class as the relative frequency of term $t$ in documents belonging to class $c$. The variation takes into account the of occurrences of term $t$ in a training documents from class $c$, including multiple occurrences. In the multinomial model, a document is an ordered sequence of word events, drawn from the same vocabulary 
V. We assume that the lengths of documents are independent of class.2 We again make a similar naive Bayes assumption: that the probability of each word event in a document is independent of the word's context and position in the document. Thus, each document di is drawn from a multinomial distribution of words with as many independent trials as the length of di. This yields the familiar "bag of words" representation for documents. Define Nit to be the count of the number of times word $w_{t}$ occurs in document $d i$. Then, the probability of a document $t$ given its class from Equation 1 is simply the multinomial distribution:

$$
P\left(d_{i} \mid c_{j} ; \theta\right)=P\left(\left|d_{i}\right|\right)\left|d_{i}\right| ! \prod_{t=1}^{|V|} \frac{P\left(w_{t} \mid c_{j} ; \theta\right)^{N_{i t}}}{N_{i t} !}
$$

The parameters of the generative component for each class are the probabilities for each word, written $\theta_{w t} \mid c_{j}=P\left(w_{t} \mid c_{j} ; \theta\right)$, where $0 \leq \theta_{w t} \mid c_{j} \leq 1$ and $\sum_{t} \theta_{w t} \mid c_{j}=1$. [Multinomial Answes]

Bernoulli Naïve Bayes is a multi-variate event model in which a document is a binary vector over the space of words. Given a vocabulary $V$, each dimension of the space $t, t \in\{1, \ldots,|\mathrm{V}|\}$, corresponds to word $\mathrm{w}_{\mathrm{t}}$ from the vocabulary. Dimension $t$ of the vector for document $d_{i}$ is written $\mathrm{B}_{\mathrm{it}}$, and is either 0 or 1 , indicating whether word $\mathrm{W}_{\mathrm{t}}$ occurs at least once in the document. With such a document representation, we make the naive Bayes assumption: that the probability of each word occurring in a document is independent of the occurrence of other words in a document. Then, the probability of a document given its class from Equation 1 is simply the product of the probability of the attribute values over all word attributes:

$$
P\left(d_{i} \mid c_{j} ; \theta\right)=\prod_{t=1}^{|V|}\left(B_{i t} P\left(w_{t} \mid c_{j} ; \theta\right)+\left(1-B_{i t}\right)\left(1-P\left(w_{t} \mid c_{j} ; \theta\right)\right)\right)
$$


Naïve Bayes, Multinomial Naïve Bayes and Bernoulli Naïve Bayes all the Bayes classifier are built in library of scikit learn. However, to apply Support vector model and Stochastic gradient descent we have to use another built in library of scikit learn which is called TfidfVectorizer. It takes the features and creates a matrix which is called bag of words. This bag of word consists of count of every single word. It assigns a weight to each word, basing on the words occurrence on the review texts. If a word occurs frequently it gets a low weight and a word occurring least frequently gets higher weight. There are two different classes for bag of words and TfidfVectorizer, which are called CountVectorizer and TfidfTransformer. These two steps are done in single step in this methodology. After assigning weights we can perform Linear SVC, NuSVC, Stochastic Gradient Descent and Logistic regression.

Mentioned earlier, the classification algorithms are to classify the dataset which consists of features and find out the accuracy of the algorithms. The classification is done in a various numbers of feature sets starting from 1000 . We have to find out the accuracy for different algorithm by gradually increasing the number of feature set. However, we have to increase our feature set number because we want to get the best accuracy for every algorithm. We have started our classification from 1000 till 12000 because the computing power could not handle large number of feature set. 
In table I. the accuracy of the classifiers along with the feature set is shown.

TABLE I. ACCURACY OF ALL THE CLASSIFIERS

\begin{tabular}{|l|l|l|l|l|l|l|l|l|l|}
\hline $\begin{array}{l}\text { Number } \\
\text { of } \\
\text { feature } \\
\text { sets }\end{array}$ & $\begin{array}{l}\text { Naïve } \\
\text { Bayes }\end{array}$ & $\begin{array}{l}\text { Bernoulli } \\
\text { Naïve } \\
\text { Bayes }\end{array}$ & $\begin{array}{l}\text { Multinomial } \\
\text { Naïve } \\
\text { Bayes }\end{array}$ & SVC & $\begin{array}{l}\text { Linear } \\
\text { SVC }\end{array}$ & NuSVC & $\begin{array}{l}\text { Logistic } \\
\text { Regression }\end{array}$ & SGD & $\begin{array}{l}\text { Random } \\
\text { Forest }\end{array}$ \\
\hline $\mathbf{1 0 0 0}$ & 69.23 & 70.25 & 69.10 & 71.26 & 70.89 & 71.36 & 70.87 & 72.25 & 74.27 \\
\hline $\mathbf{1 5 0 0}$ & 70.36 & 71.31 & 70.39 & 73.21 & 74.25 & 73.15 & 72.58 & 71.63 & 75.93 \\
\hline $\mathbf{2 0 0 0}$ & 71.32 & 70.69 & 72.15 & 74.34 & 75.21 & 75.32 & 73.36 & 74.35 & 76.15 \\
\hline $\mathbf{2 5 0 0}$ & 75.36 & 74.36 & 75.31 & 78.36 & 79.32 & 78.17 & 75.15 & 76.36 & 77.36 \\
\hline $\mathbf{3 0 0 0}$ & 78.36 & 77.35 & 78.14 & 80.76 & 80.29 & 80.21 & 79.01 & 78.12 & 78.48 \\
\hline $\mathbf{5 0 0 0}$ & 80.49 & 81.01 & 81.25 & 83.12 & 84.31 & 82.36 & 82.14 & 81.45 & 82.96 \\
\hline $\mathbf{6 0 0 0}$ & 81.15 & 83.25 & 83.14 & 84.25 & 85.15 & 84.12 & 83.36 & 82.95 & 85.35 \\
\hline $\mathbf{7 0 0 0}$ & 82.25 & 84.11 & 84.16 & 85.85 & 86.36 & 85.36 & 86.16 & 84.01 & 87.16 \\
\hline $\mathbf{8 0 0 0}$ & 83.89 & 86.36 & 86.26 & 87.01 & 86.89 & 87.15 & 88.09 & 85.98 & 89.15 \\
\hline $\mathbf{1 0 0 0 0}$ & 85.99 & 88.29 & 88.81 & 89.36 & 88.59 & 89.15 & 89.95 & 88.39 & 92.63 \\
\hline $\mathbf{1 2 0 0 0}$ & 87.68 & 90.15 & 91.02 & 90.14 & 89.98 & 90.26 & 90.78 & 89.91 & 94.25 \\
\hline
\end{tabular}

In figure 18 we show the graphical representation of the classifiers and their accuracy. We used a python library named matplotlib for plotting the data. This library is an open source library which can plot any data frames into line chart, bar chart, scatter diagrams and many more. 


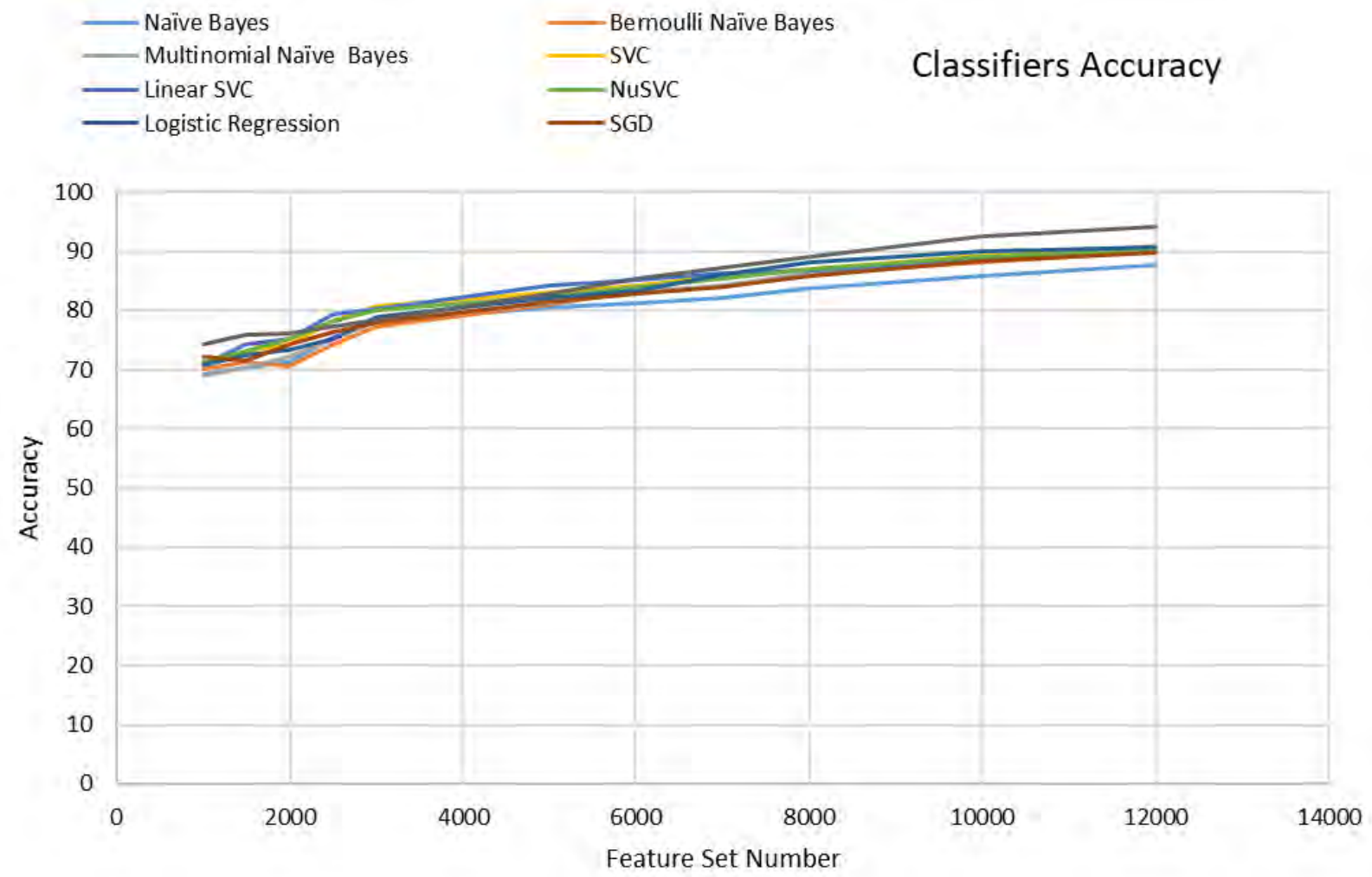

Fig 18. Accuracy of all classifiers in terms of number of feature set

\subsection{SENTIMENT POLARITY OF EACH FEATURE AND THE REVIEW}

Sentiment analysis is the process of determining the polarity or emotion behind some series of words or even a sentence. In this research we have already done data selection, training algorithms, classifying algorithms. We have already preprocessed the dataset and selected the specifications of the mobile sets for our research. Python and Scikit learn which is a machine learning library of python and NLTK which is a language processing tool widely used for the sentiment analysis and text polarity measurement is used in our research. As we already have done the classification which provided us with different accuracy for different algorithms, we choose the best algorithms for the sentiment analysis. However, we have applied all the 
classifiers individually but for the best result we have created a combination of all the classifying algorithms. This combined classifier provides us the best possible result for the pre-processed data. As mentioned earlier, we separated all the sentences of the review text according to the specification or feature of the mobile. As an example, if there is a review mentioning multiple features of the phones followed by their review we picked that sentence and we applied sentiment analysis on that sentence. In figure 19 selection of sentence process is shown in a pictorial view.

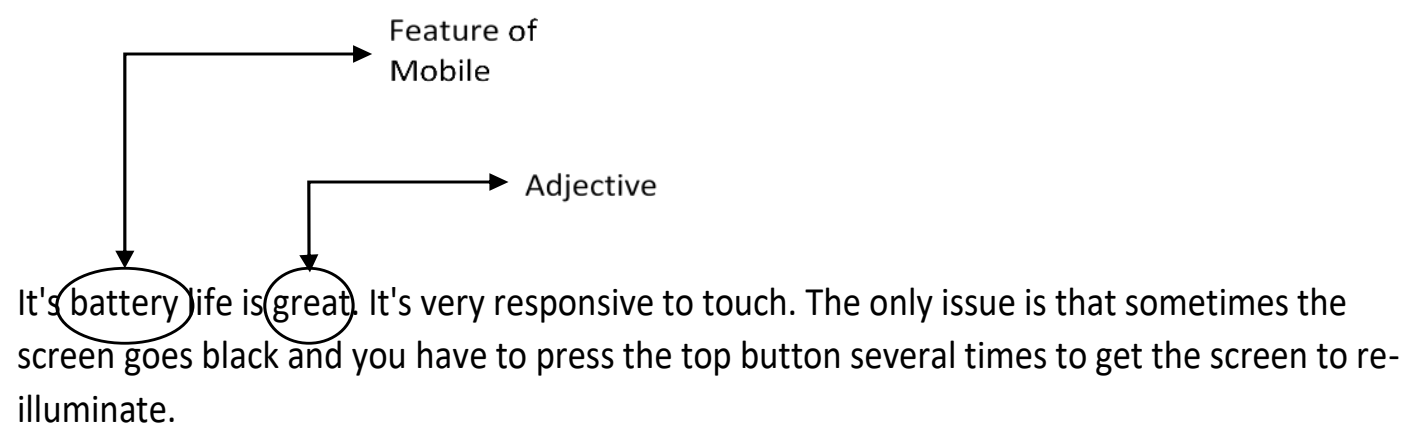

Fig. 19. Selection of Adjective and Specification (feature)

After the selection of text, we specifically applied sentiment analysis with NLTK in the adjectives or the words which can determine polarity. As an example, "Nokia is the brand that can be used in telephone, good price, excellent team, was a gift to my daughter and she liked it, easy to use and very affordable price, I recommend it." this review has one big sentence with a lot of features or specification and their classification. In figure 20 we applied sentiment analysis on this review and the result of it shows, 
C: \Python36lpython.exe C:/Users/kafia/PycharmProjects/ThesisFinalVersion/Feature.py

<-- The Review Sentence --->

Nokia is the brand that can be used in telephone, good price, excellent team, was a gift to my daughter and she liked it, easy to use and very affordable price, I recommend it.

<-- Applying Sentiment Analysis -->

Positive

Confidence Level : 98.99

Fig. 20. Result of Sentiment Analysis

As mentioned earlier we are applying sentiment analysis on the features and we want to rate the feature by finding out their polarity and the polarity score. However, to find out the polarity of all the features we applied pos tagging on the review sentence and collected the adjective which normally is the identifier of positive or negative word. For finding the adjective we used regular expression. There is a built in 're' library which is used for regular expression finding. As an example, "Nokia is the brand that can be used in telephone, good price, excellent team, was a gift to my daughter and she liked it, easy to use and very affordable price, I recommend it" we applied pos tagging which gives a result shown in figure 21 .

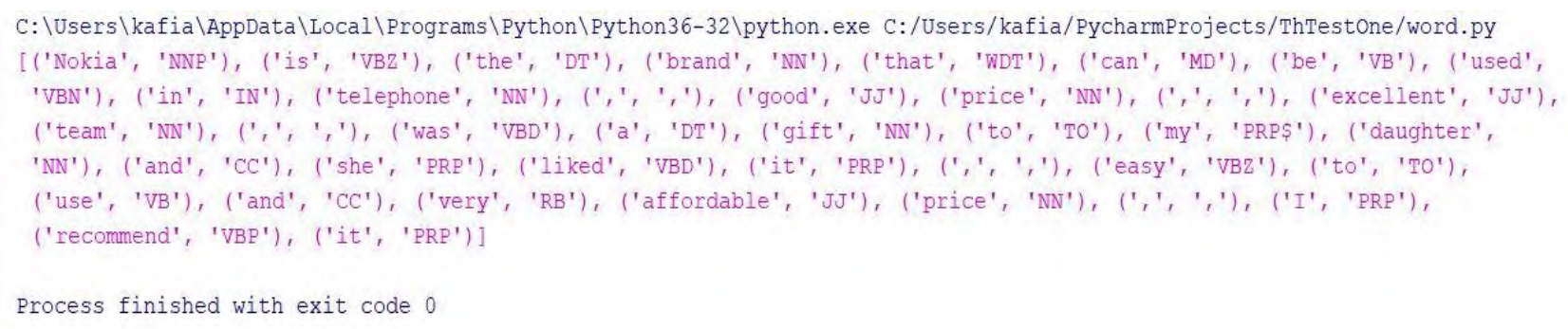

Fig. 21. Result of Pos tagging to the review

After pos tagging we applied 're' to find out the adjectives from the words. The 'JJ' tag stands for adjective in figure 21 , also we have to find out the feature which 
corresponds to the adjective. So, we search for a feature in a sentence and we also apply 're' to find out the adjective in the same sentence. The adjective will always be before or after the noun. Thus, we find the noun corresponding which relates with the adjective and select those two to apply sentiment analysis on the adjective. We store the name of the specification or feature and the adjective to a two-dimensional array. Now we apply sentiment analysis using NLTK to adjective and show the desired result and store the result in an array with the same index of the two dimensional array. Figure 22 shows the result of feature-based sentiment analysis.

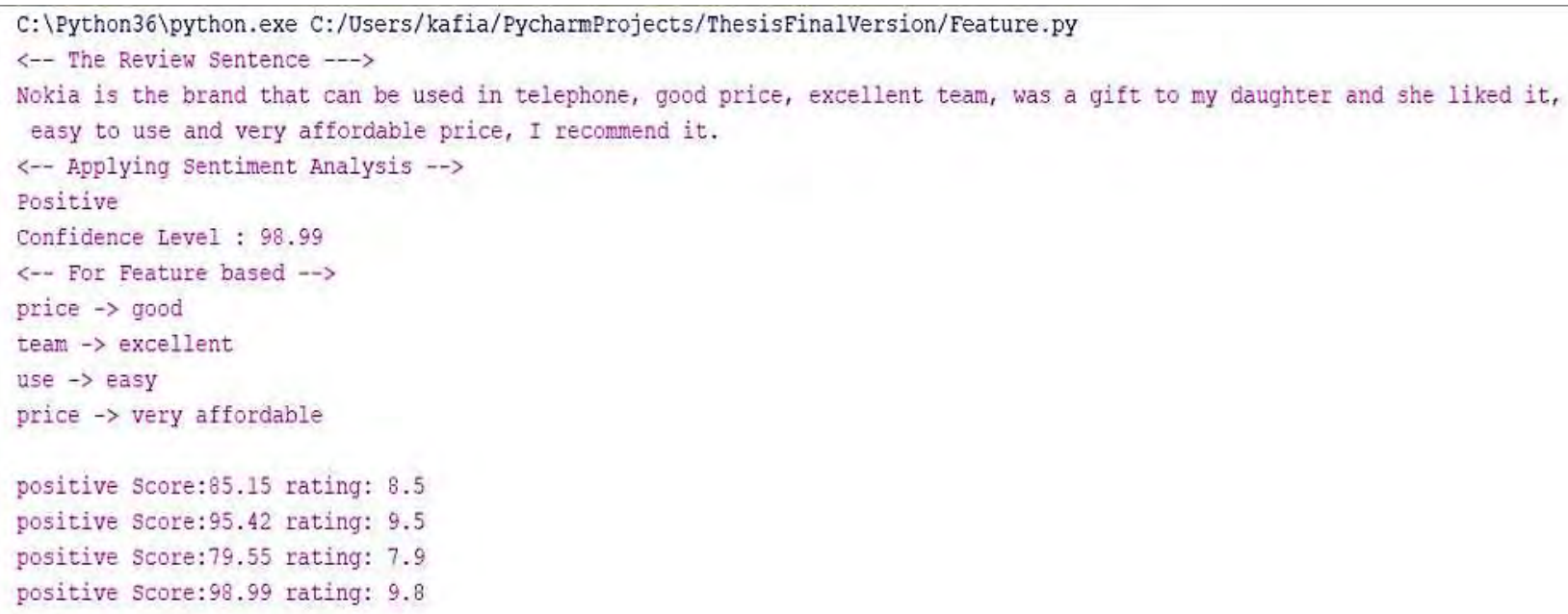

Fig. 22. Result of Feature based Sentiment analysis

\subsection{RATING THE MOBILE PHONE}

The last step of our research is to provide a rating of the mobile phone according to the review and polarity. In previous step we found out the polarity of each feature and the score of the polarity or confidence of polarity which starts from 0 to 100 . However, to rate the polarity we divided our polarity score by 10 and the result we get is the rating. So, the rating will always be from 0 to 10 . The equation of rating is, 


$$
\text { rating }=\frac{\text { polarity score }}{10}
$$

This gives us the individual rating of each feature. However, to find out the rating of the product or mobile phone we have to find the average rating of each feature and that will give us the rating of the desired mobile phone.

$$
\sum_{i}^{n} \frac{\text { rating of each feature }}{n}
$$

In figure 23 the result of the example is shown, which consists of the final result of sentiment analysis.

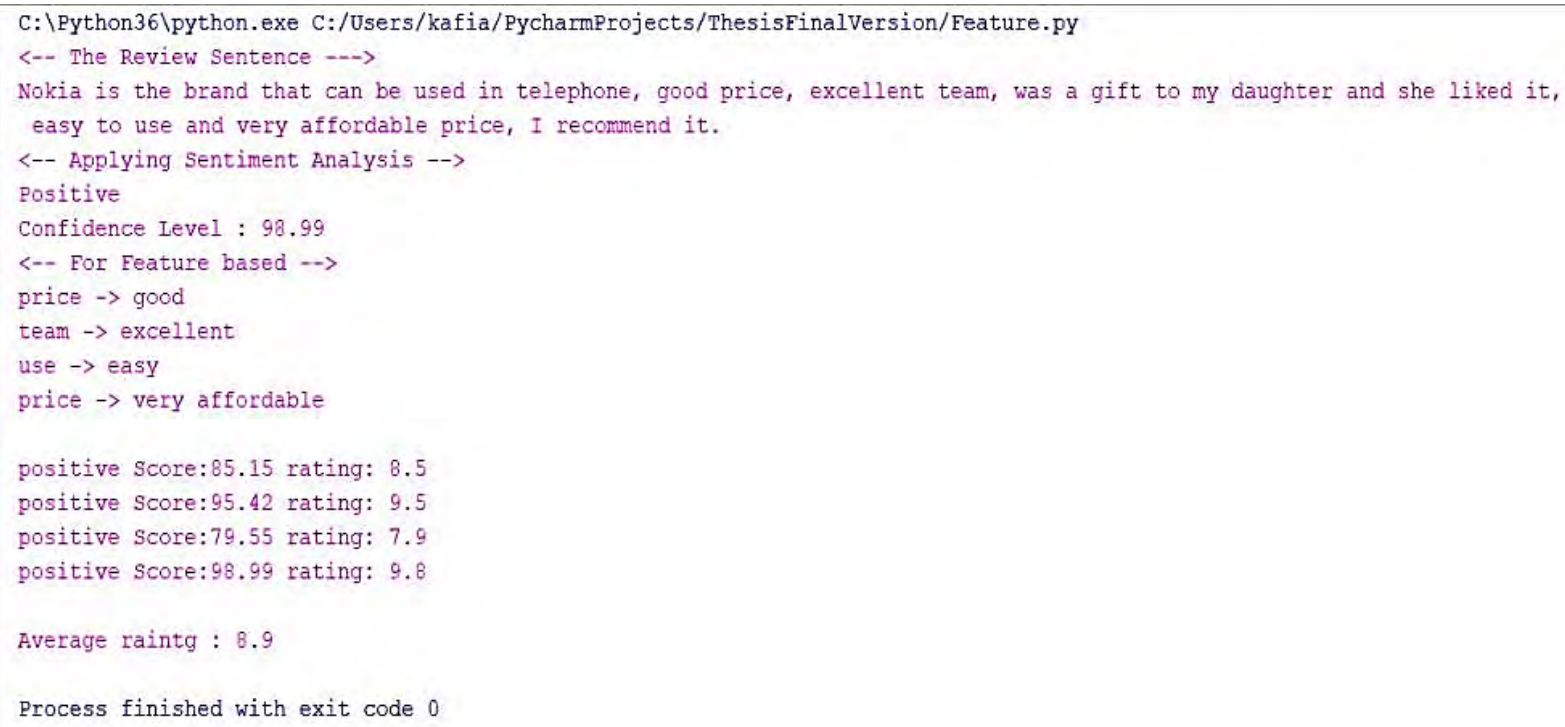

Fig. 23. Final result of sentiment analysis

However, we also applied another way to find the rating. As mentioned earlier the model stores the feature of a mobile phone along with the adjective in a two dimensional array and the result of the sentiment in an array with the same index. For, each model of mobile we searched for the number of features in our two 
dimensional array and counted them. The number of positives and negatives is also counted. We find the percentage of positive and divided that percentage by 10 to give a rating which is eventually divided by 2 to get the rating in the rage of 0 to 5 .

The framework we applied in our research seems very convenient in terms of mobile selection for customers. This methodology will help the customers to evaluate any mobile phone based on public rating. 


\section{CHAPTER 4 EXPERIMENTAL SETUP AND RESULT ANALYSIS}

The research of sentiment analysis and machine learning always requires heavy computing power. In our research, we had to use Keras and Tensorflow as a backend engine which actually supports the Scikit learn library. Scikit learn is basically run by Keras. As mentioned earlier there are multiple algorithms in our model and the dataset is also very large. First of all we have to initialize the data.

\subsection{DATASET PREPARATION}

As mentioned earlier the dataset we collected is very large, so we used panda to minimize our data by creating another dataset with less number of data. Next, we had to split our dataset into train set and test set.

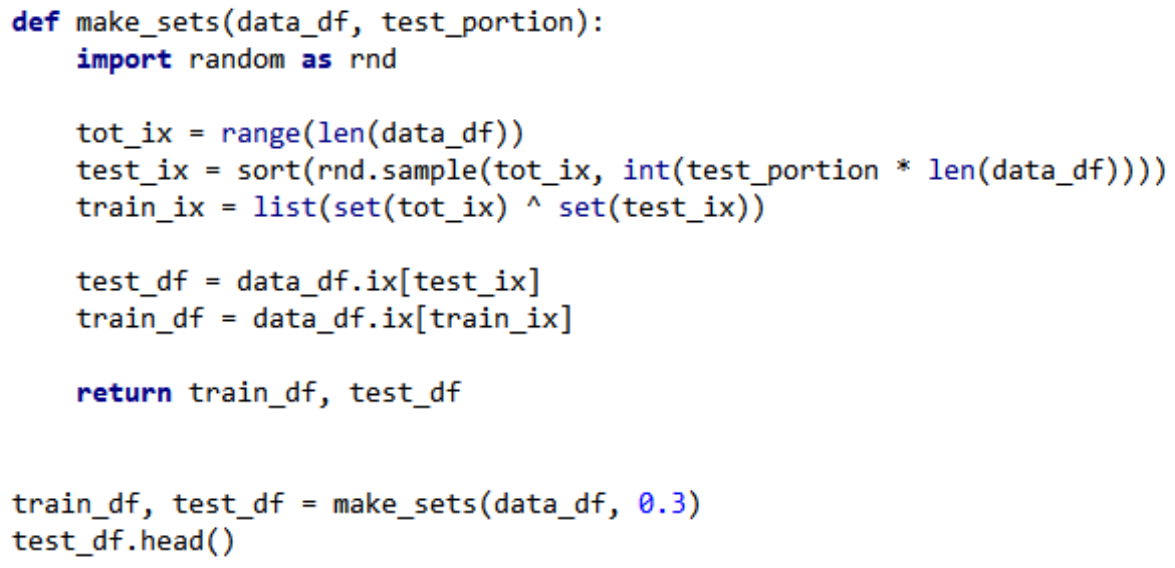

Fig. 24. Dataset splitting

Then we train our algorithms using the Sklearn library. Sklearn has libraries for all the algorithms and we have to just assign the train set to our algorithms. 


\subsection{CLASSIFICATION AND RESULT}

As we mentioned in chapter 3, we had to classify the dataset with all the classifiers and get the accuracy. The accuracy result indicates, the higher the number of feature set the higher the accuracy. But due to limitations in computing power higher number of dataset is not used in our research.

However, we applied classifiers and also their types to classify the feature set and found out the accuracy of the classifiers. Naïve Bayes has two different sub classes and in in figure 25 the comparison between these classifiers are shown where we classified 5200 feature set with these classifiers and got three different accuracy.

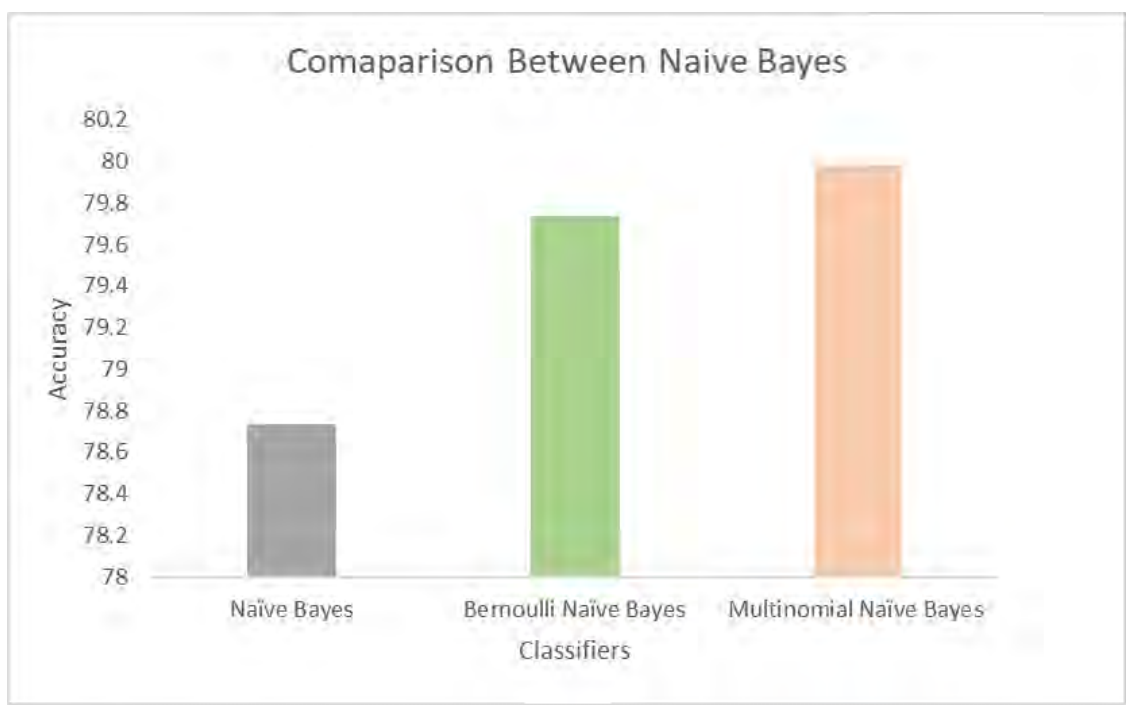

Fig. 25. Comparison between Naïve Bayes classifiers

We also used SVC, linear SVC and NuSVC which performs multi class classification on the dataset. In our research we used these three algorithms to classify 5200 classifiers, which is given in figure 26 . 


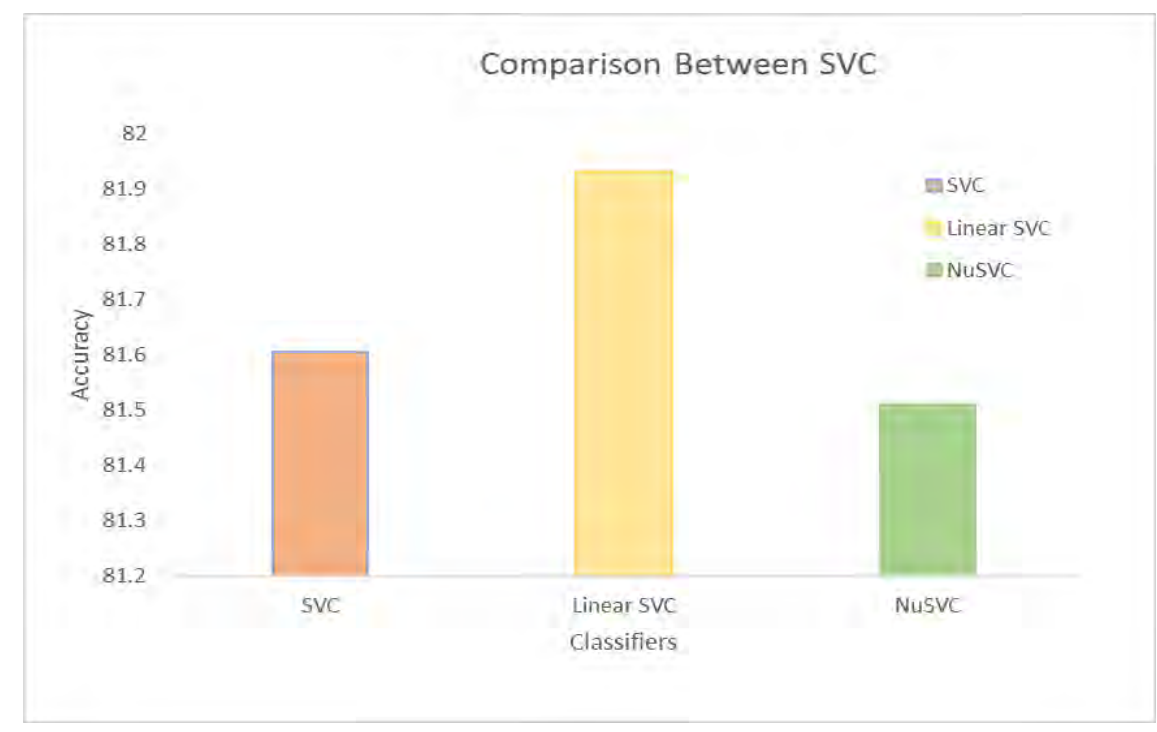

Fig. 26. Comparison between Svc classifier's

We also applied Stochastic Gradient Descent, Logistic Regression which are part of linear regression and random forest, which is very known for text classification. The accuracy we got after applying these classifiers on 5200 features are shown in figure 27.

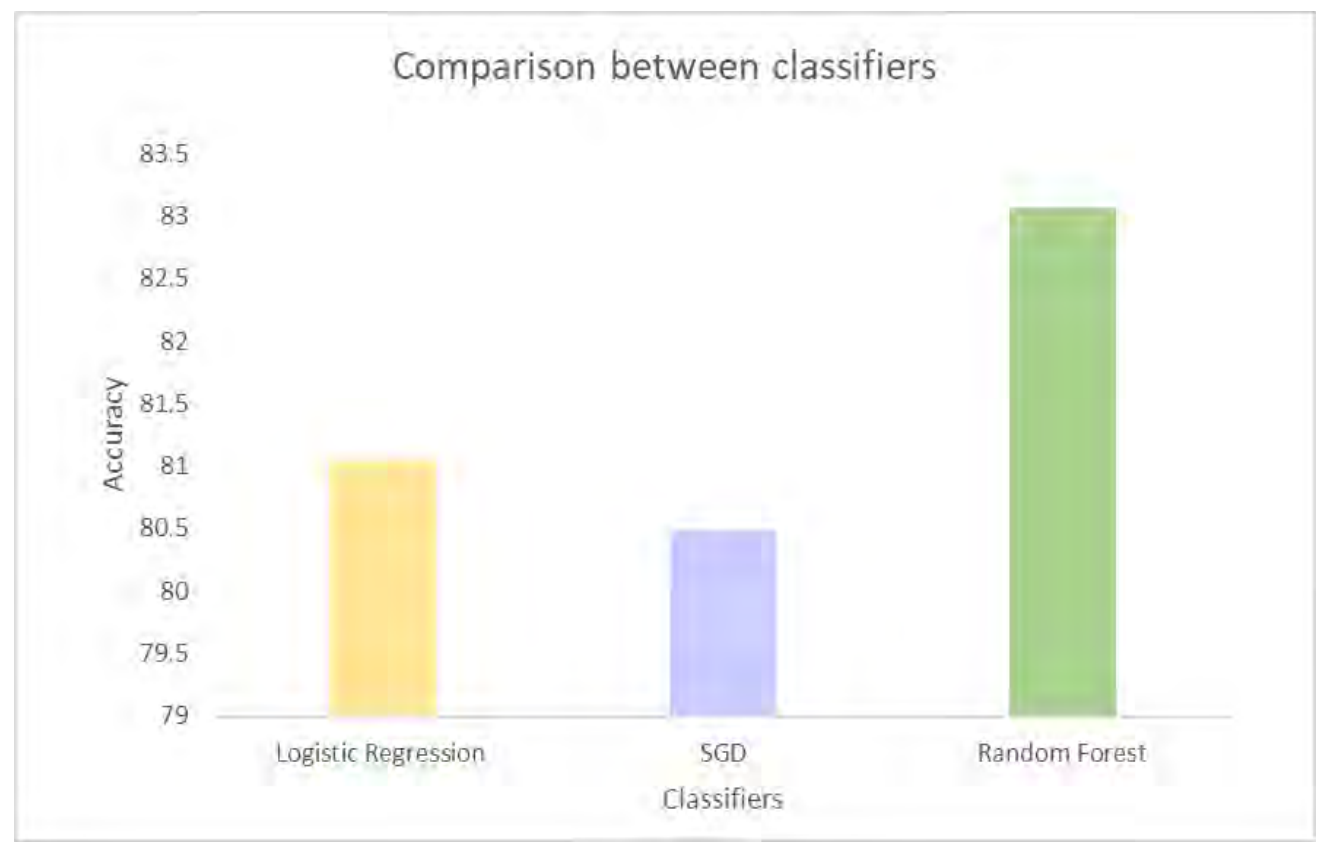

Fig. 27. Comparison between classifiers 
After all the classification we see that linear SVC and Random forest gave the best accuracy. In 5200 feature sets these two classifiers scored the most accuracy of $82 \%$ and $83 \%$. The accuracy of the classification increases when we apply in large number of feature sets.

However, to get the best result from any textual classification we used a voted classifier which is a built in method in Scikit learn library. In this way, we can use the best possible result from each classifier and create a classifier which will be a merged classifier. The merged classifier will represent all the classifiers and give a better result. Moreover, after applying the voted classifier on 5200 features set we got an accuracy of $86.34 \%$ which is very good in terms of small scale dataset. In figure 28 we showed the comparison between all the classifiers and voted classifier.

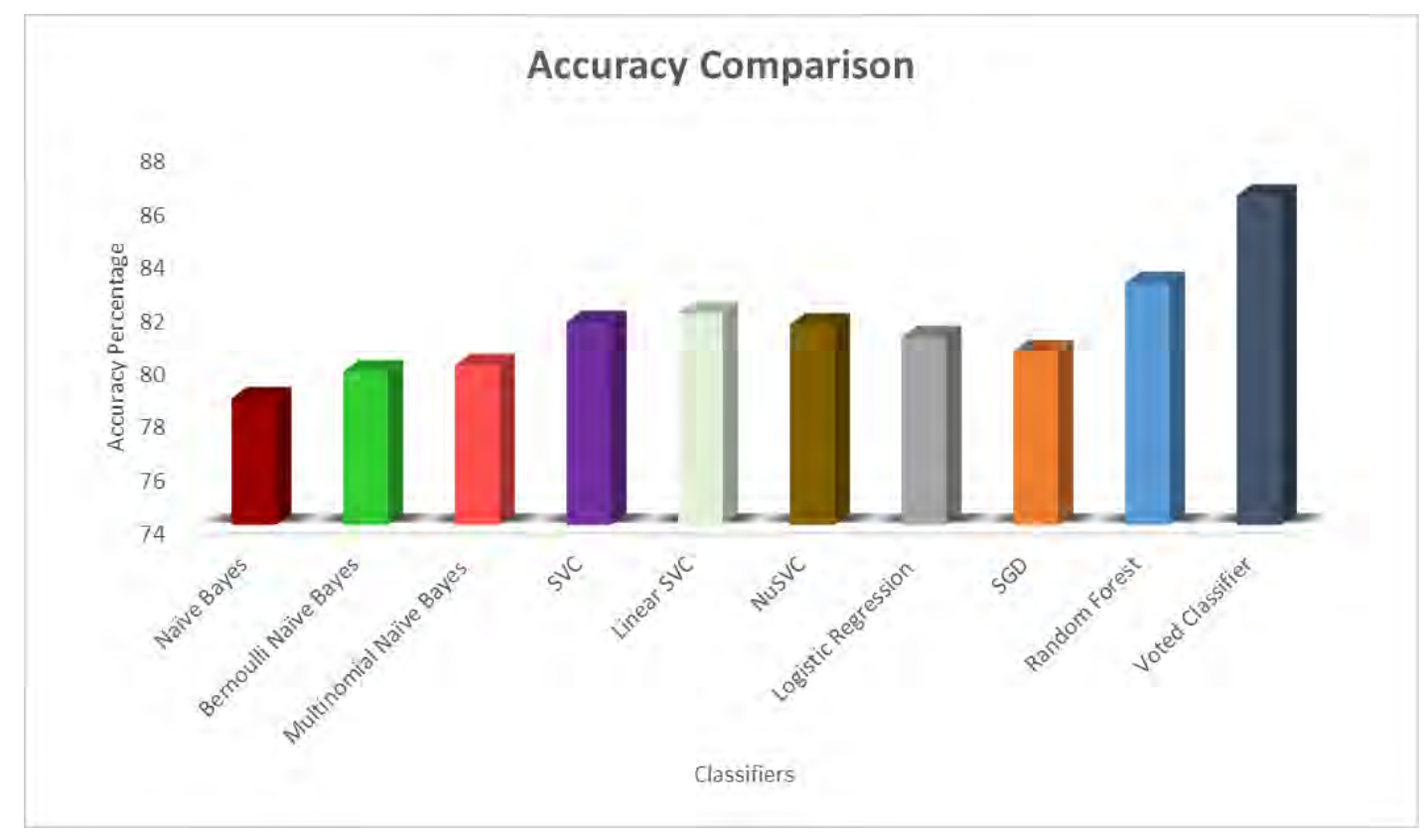

Fig. 28. Accuracy Comparison between all classifiers and voted classifier 


\subsection{SENTIMENT ANALYSIS ON REVIEW}

Sentiment analysis is the process of finding out emotion of a textual expression. In this research we have pre-processed our dataset, trained classifier, tested the classifiers to find out the accuracy. For sentiment analysis we have used Scikit learn library, NLTK and Python programming language. However, to find out the polarity of a review we have done text preprocessing, cleaning etc. In chapter 3 we have discussed how to find out the polarity of a review sentence. We applied the voted classifier to find out the accuracy of any review sentence. As our model is for finding out the specific review for each and every specification of a mobile phone we applied sentiment analysis also on specific words. As an example, "Nokia is the brand that can be used in telephone, good price, excellent team, was a gift to my daughter and she liked it, easy to use and very affordable price, I recommend it." is a review given by a customer of Nokia phone. The model first finds out the polarity of the sentence and then it also searches for the features or specification of a mobile phone and their corresponding adjective which actually determines the sentiment of that review. In figure 29 the outcome of a sentiment analysis is shown.

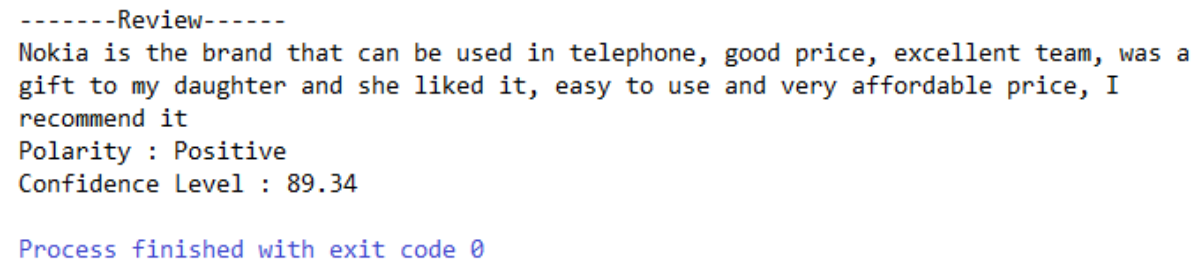

Fig. 29. Result of sentiment analysis

Moreover, our model also determines the rating of a mobile set by calculating the mean value of all ratings of that particular mobile phone. In chapter 3 we mentioned the process briefly. However, from our research data we found some comparisons 
between some mobile set models of different brands. In figure 30 we show a graphical representation of the ratings between the mobile phones.

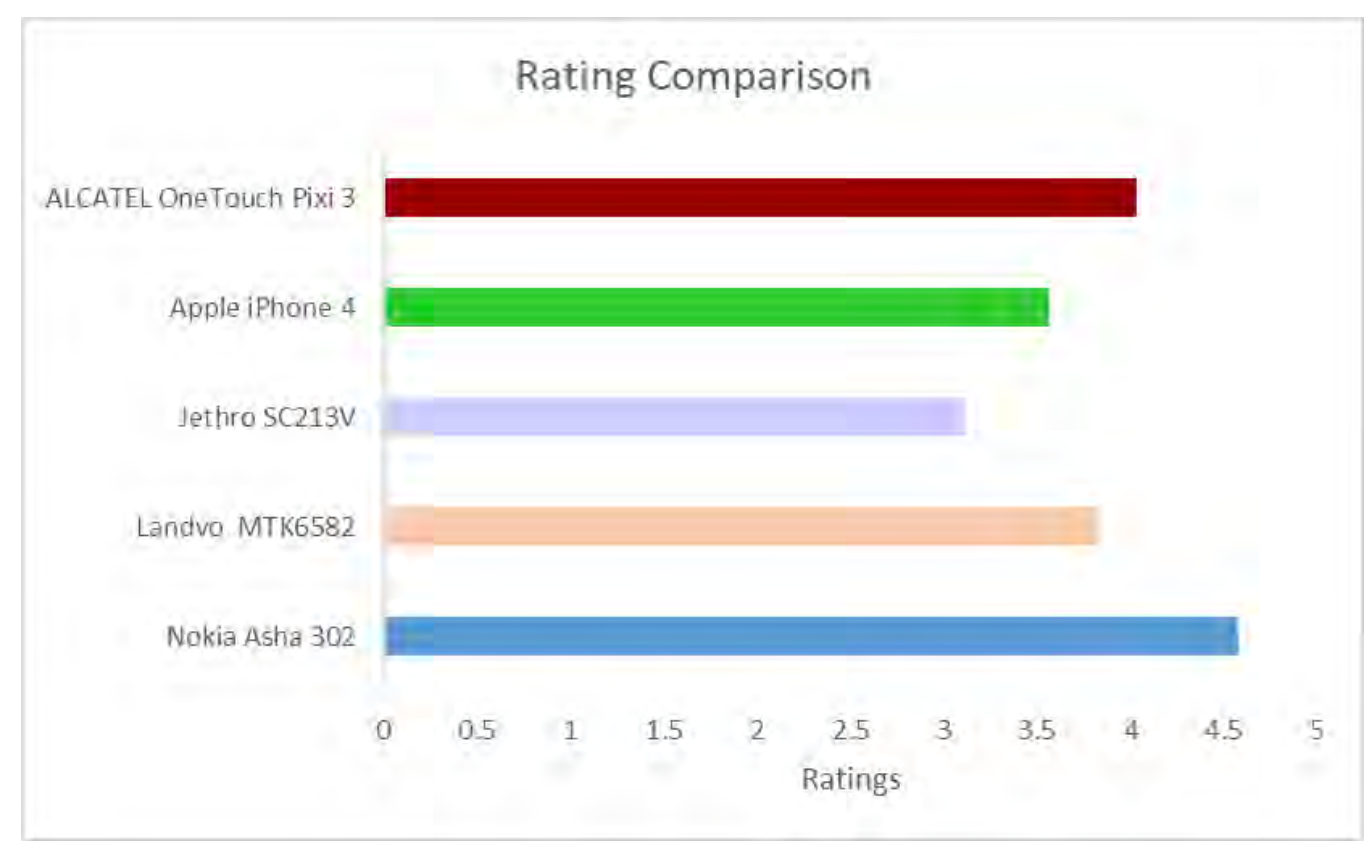

Fig. 30. Rating comparison between different models

Moreover, the model we prepared in this research can also give specific review ratings which actually is very helpful for the customers. This methodology can be applied in any online shops with reviews. In table 4.3.1 the ratings of all the specification found for iPhone 4 16GB is given. 
TABLE II. IPHONE 4'S SPECIFICATIONS RATING

\begin{tabular}{|l|l|}
\hline Specification & Rating \\
\hline Camera & 4.6 \\
\hline Outlook & 4.0 \\
\hline RAM & 4.5 \\
\hline Storage & 5 \\
\hline Display & 4.8 \\
\hline Battery & 4.3 \\
\hline App Store & 2 \\
\hline IOS & 3.6 \\
\hline
\end{tabular}

This table shows the specific reviews which we found out applying our methodology on the review comments of customers. 


\section{CHAPTER 5 \\ CONCLUSION AND FUTURE WORK}

\subsection{CONCLUSION}

In today's world, uses of sentiment analysis can play a significant role to understand people's opinion automatically and use that to improve certain product or service efficiently. This can be a field of interest for both researchers and entrepreneurs. In this research, the model that is proposed analyses the sentiment of customers review of mobile phones which is collected from Amazon and rate them according to their features. Naïve Bayes, Support Vector Machine, Logistic Regression, Stochastic Descent algorithm are used in this model and accuracy of their performance are compared which showed that SVC and Random forest perform better with greater accuracy. Using multiple algorithm helped to understand which algorithm is more suitable for this system. However, using the average classifier or combined classifier gives better accuracy always. Ratings of the product is given based on the average polarity obtained which can help the customer to know about certain products or services and take decisions accordingly. From this research work it is found that, the cleaner the data, the better the accuracy of the result and the more the feature set the more the accuracy. In future, this model can be implemented as web application so that while a customer is willing to buy a certain product, they can easily make a decision just by looking at the ratings.

\subsection{FUTURE WORK}

In this research., a model was proposed to rate mobile phones based on public opinion. For future work we want to analyze the reviews of mobile phones on multiplatform, meaning for a mobile phone review that is available on more than 
one platform we want compare how user opinion varies for a mobile phone on different platforms and also show rating for each version of the phone. Along with that we also want to find a correlation between user opinion and sales number of a mobile phone to find out if there is a relation between user opinion of a mobile phone that is shifting from time to time and sales number of the mobile in that given time. So for business persons or industry wise we can rely on this model. In the future, we want to improve this model and bring to a stage where it not only works flawlessly with mobile phone reviews but works with other art form reviews for example movies, TV shows, books, computers etc. Along with that we also want to provide a way to make our model more user friendly.

We also want to improve the algorithms that we have used in this research. In future, our goal is to apply unsupervised machine learning in the model. We want to apply neural network, CNN algorithms to find out the sentiments of a review. This model can be applied to any of the systems.

We are looking forward to make decision making process easier when it comes to selecting mobile phones for the users. Our belief is that we can make this model user friendly and accurate enough for users to use this on regular basis for judging mobile phones and save their time from looking to different sites to find good reviews. 


\section{REFERENCE}

1. K. Wójcik and J. Tuchowski, "Feature based sentiment analysis", presented at the 3rd International Scientific Conference on Contemporar Issues in Economics, Business and Management EBM, 2014.

2. R. Safrin, K.R.Sharmila, T.S.S. Subangi and E.A. Vimal, "Sentiment Analysis on Online Product Review", International Reseach Journal of Engineering and Technology (IRJET), vol.04, issue.04. 2017.

3. A. Rane and Dr.A.Kumar, "Sentiment Classification System of Twitter Data for US Airline Service Analysis", presented in 42nd IEEE International Conference on Computer Software \& Application, 2018.

4. M.S.Wai and S.S.Aung, "Simultaneous Opinion Lexicon Expansion and Product Feature Extraction", Third Seminar, University of Computer Studies, Mandalay, 10.13140/RG.2.2.28250.44489, 2018.

5. X. Fang and J. Zhan, "Sentiment Analysis Using Product Review Data", Journal of Big Data, 2. 10.1186/s40537-015-0015-2, 2015.

6. Z. Jianqiang and G. Xiaolin, "Deep Convolution Neural Networks for Twitter Sentiment Analysis", IEEE, 2017.

7. R.Rafeek and R. Remya, "Detecting contextual word polarity using aspect based sentiment analysis and logistic regression", 2017 IEEE International Conference on Smart Technologies and Management for Computing, Communication, Controls, Energy and Materials (ICSTM),2017.

8. B.Wagh,Prof.J.V.Shinde and Prof.P.A.Kale, "A Twitter Sentiment Analysis 
Using NLTK and Machine Learning Techniques",International Journal of Emerging Research in Management \&Technology ISSN: 2278-9359 (Volume-6, Issue-12),2017.

9. S.S.Kamble and Prof.A.R.Itkikar, "Study of supervised machine learning approaches for sentiment analysis", International Research Journal of Engineering and Technology (IRJET) (Volume: 05 Issue: 04),2018.

10. S.B.Kim, K.S.Han and H.C.Rim, "Some Effective Techniques for Naive Bayes Text Classification," IEEE Transactions on Knowledge and Data Engineering (Volume: 18, Issue: 11, Nov. 2006 ), 2006.

11. R. Varghese and M Jayasree, "Aspect based Sentiment Analysis using support vector machine classifier," 2013 International Conference on Advances in Computing, Communications and Informatics (ICACCI), 2013.

12.Y.E.Cakra and B.D.Trisedya, "Stock price prediction using linear regression based on sentiment analysis," 2015 International Conference on Advanced Computer Science and Information Systems (ICACSIS), 2015.

13. R.Rafeek and R. Remya, "Detecting contextual word polarity using aspect based sentiment analysis and logistic regression," 2017 IEEE International Conference on Smart Technologies and Management for Computing, Communication, Controls, Energy and Materials (ICSTM),2017.

14. F.Kabir, S.Siddique, M.R.A.Kotwal and M.N.Huda, "Bangla text document categorization using Stochastic Gradient Descent (SGD) classifier," 2015 International Conference on Cognitive Computing and Information Processing(CCIP),2015. 